\title{
PREPRINT
}

\section{IncRNA Neat1 drives neuronal histone methylation and age-related memory impairments}

\author{
Anderson A. Butler ${ }^{1}$, Daniel R. Johnston1 ${ }^{1}$, Simranjit Kaur ${ }^{1}$, and Farah D. Lubin ${ }^{1 *}$ \\ 1Department of Neurobiology, University of Alabama at Birmingham, Birmingham, AL 35294, USA. \\ *Author for correspondence (flubin@uab.edu)
}

\begin{abstract}
Histone methylation is critical for the formation and maintenance of long-term memories. Long noncoding RNAs (IncRNAs) are regulators of histone methyltransferases and other chromatin modifying enzymes (CMEs). We investigated how IncRNA Neat1-mediated histone methylation contributes to hippocampusdependent long-term memory formation, using a combination of transcriptomics, RNA binding protein immunoprecipitation, CRISPR mediated gene activation, and behavioral approaches. Suppression of the IncRNA Neat1 revealed widespread changes in gene transcription as well as perturbations of histone 3 lysine 9 dimethylation (H3K9me2), a repressive histone modification mark that is dysregulated in the aging hippocampus. We identified a Neat1-dependent mechanism of transcriptional repression via H3K9me2 at the $c$-Fos promoter corresponding with observed changes in c-Fos mRNA levels. Overexpression of hippocampal Neat1 via CRISPRa is sufficient to impair memory formation in young adults, recapitulating observed memory deficits in old adults, while Neat1 suppression in both young and old adult mice improves memory. These results suggest that IncRNA Neat1 is a potent epigenetic regulator of hippocampus-dependent long-term memory formation.
\end{abstract}

\section{INTRODUCTION}

While recent efforts have characterized thousands of IncRNAs in the human and mammalian genome, few IncRNAs are as wellstudied, as the human Nuclear-Enriched Abundant Transcript 1 (NEAT1). NEAT1 is evolutionarily conserved between rodents and humans, particularly within the 5' region of the transcript ${ }^{1}$. Multiple isoforms of NEAT1 exist in rodents and in humans, with the longer of the major isoforms proving essential for phase separation and induction of nuclear paraspeckle assembly 2,3, while the shorter NEAT1 transcripts do not appear to be a major regulator of paraspeckle formation ${ }^{4}$. Recent studies have characterized a number of molecular pathways by which NEAT1 regulates the epigenome, including both paraspeckle-dependent sequestration of transcription factors, as well as paraspeckle-independent roles for NEAT1 in transcriptional regulation via scaffolding of CMEs ${ }^{5-}$ 7. Additionally, NEAT1 itself has been observed to bind numerous genomic loci and to effect regulation of transcription ${ }^{8,9}$.

Research on the human NEAT1 has been largely focused on its role as an oncogene in various cancers (as reviewed previously ${ }^{10}$ ), which occurs largely through its regulation of epigenetic mechanisms. However, the rodent homolog Neat1 is also

Butler et al., 2019 upregulated in the hippocampus of aging mice ${ }^{11}$ and has recently been linked to multiple cognitive and neurodegenerative disorders, including schizophrenia12, Huntington's Disease ${ }^{13}$, Parkinson's Disease ${ }^{14,15}$, Alzheimer's Disease ${ }^{16}$, and epilepsy ${ }^{17,18}$. Furthermore, recent evidence suggests that Neat1 may play a role in neuroplasticity 17 ; however, despite such extensive health relevance, the role of Neat 1 in epigenetic regulation of genes within hippocampal neurons, particularly during long-term memory formation. We used RNA-sequencing, CRISPR mediated gene activation (CRISPRa), and memory tests to investigate the functional role of IncRNA Neat 1 in gene expression dynamics and the role that age-related changes in Neat 1 expression might play in memory deficits in older adults.

\section{RESULTS}

\section{Expression of the long noncoding RNA NEAT1 is restricted in human CNS tissues}

Expression of NEAT1 is abundant in many cultured cell lines including those characterized in the ENCODE project ${ }^{19}$ (Fig. 1A). However, we observed that in contrast to the abundant expression of NEAT1 observed in most tissues, the human central nervous system (CNS) as a whole, as well as the hippocampus (outlined in red, Figs. 1B-C) express minimal quantities of NEAT120,21. Unsupervised hierarchical clustering based on tissue expression of 
bioRxiv preprint doi: https://doi.org/10.1101/531707; this version posted January 30, 2019. The copyright holder for this preprint (which was not certified by peer review) is the author/funder. All rights reserved. No reuse allowed without permission.

A Gene Annotation

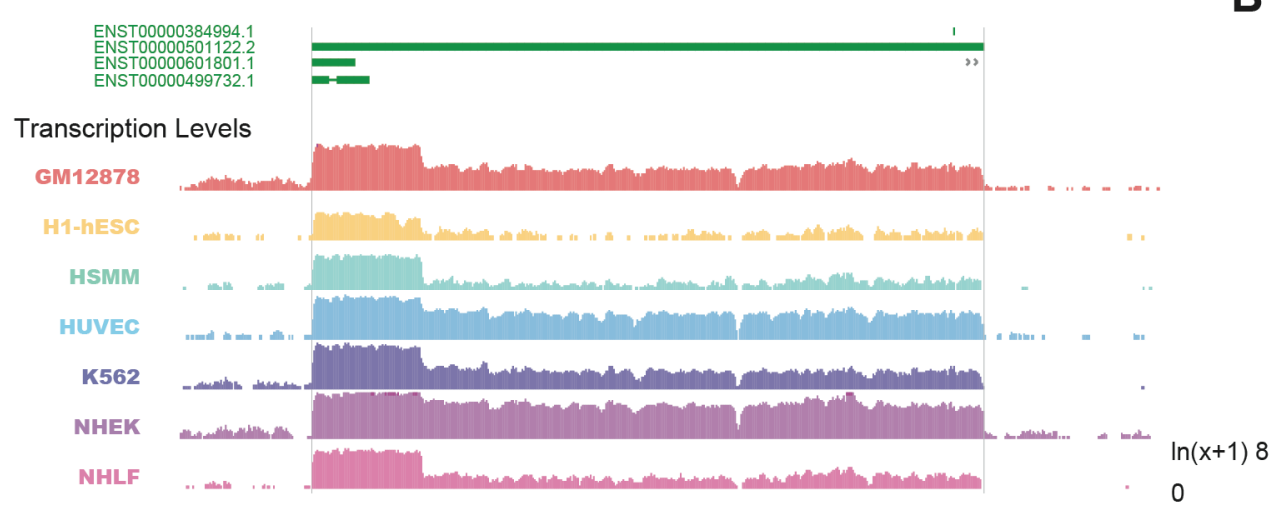

B TPM

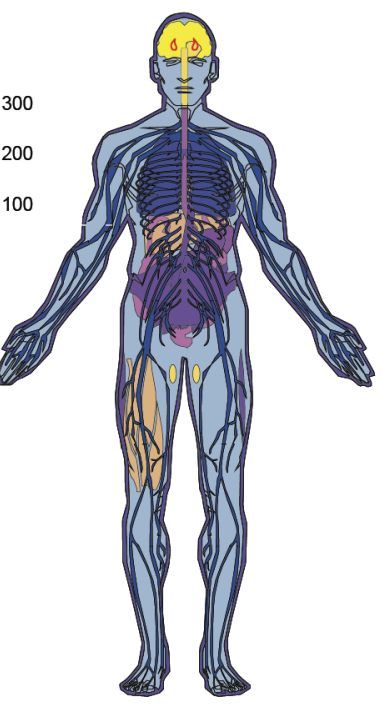

C
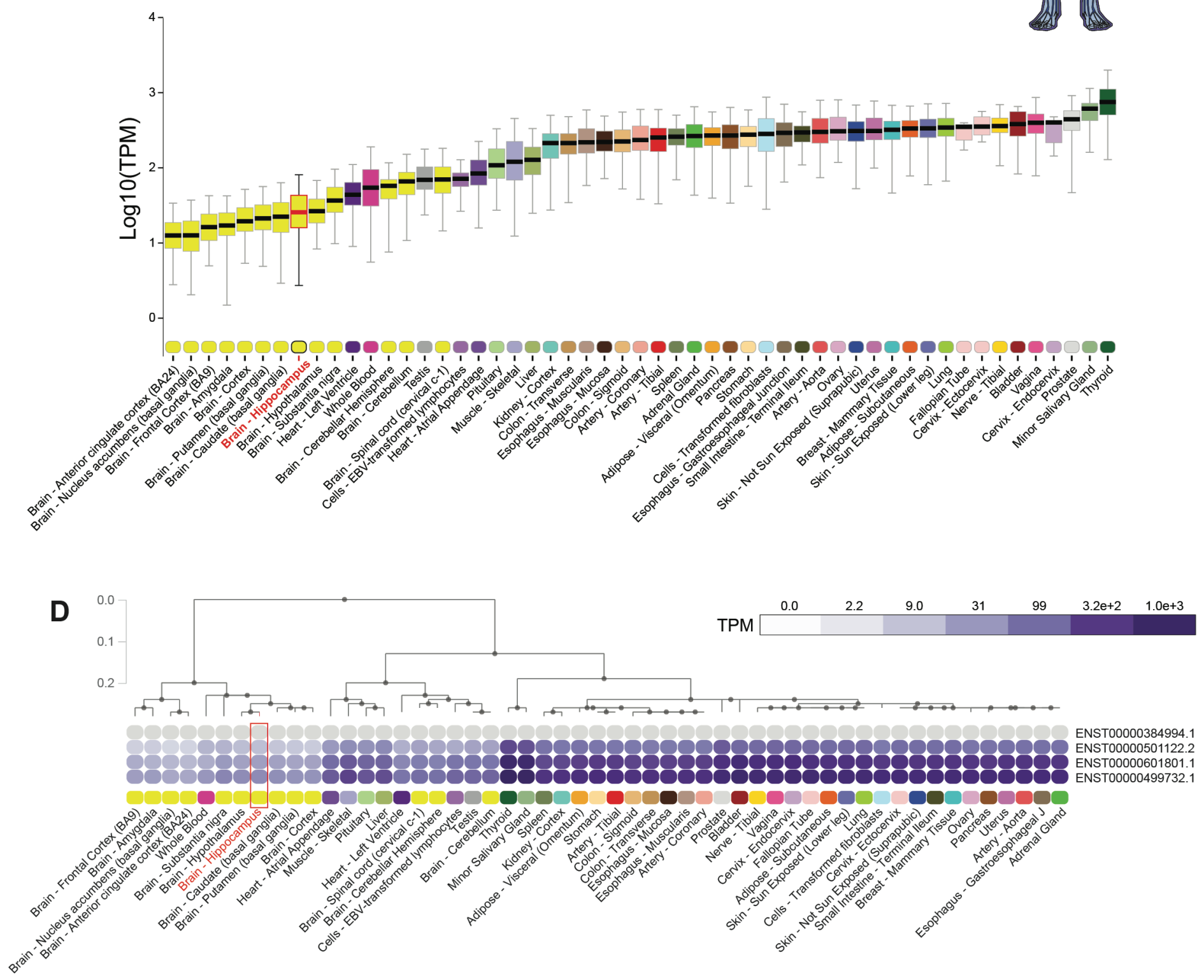
Figure 1. Restricted expression of IncRNA NEAT1 in human CNS tissues. (A) University of California, Santa Cruz (UCSC) Genome browser track export showing expression of NEAT1 in seven cell types from ENCODE. (B) Human body plot illustrating the expression of NEAT1 in 53 human tissues from the GTEx project, values shown are the median transcripts per million (TPM) values by tissue, hippocampus outlined in red. (C) Bar plots showing median, upper quartile, and lower quartile expression of the NEAT1 gene (ENSG00000245532.4) in 53 human tissues from the GTEx project; hippocampal expression outlined in red. (D) Hierarchical clustering of NEAT1 based on transcript isoform level expression in 53 human tissues from the GTEx project. Dendrogram scale shows cluster distance. Expression values displayed in the heatmap are the median expression values in TPM for each isoform in each tissue.

NEAT1 supports this observation, as CNS tissues segregate cleanly when sorted based on NEAT1 transcript expression (Fig. 1D, Supplemental Figs. 1A-B).

Examination of single-cell RNA-seq data from resected human CNS tissue and glioblastoma 22 further suggests that expression of NEAT1 within CNS cells is restricted in neurons, while other cell types including astrocytes, oligodendrocytes, and vascular cells express NEAT1 at higher levels. (Supplemental Figs. 1C-D). This is in contrast to the neighboring IncRNA transcript MALAT1 which appears to be ubiquitously expressed at high levels in all CNS cell types (Supplemental Fig. 1E). Given the growing body of literature that has noted overexpression of NEAT1 in the aging brain 23,24 , as well as the established role of NEAT1 as a regulator of epigenetic mechanisms, and the recentlydescribed role of NEAT1 in cognitive disorders such as schizophrenia ${ }^{12}$, we sought to further investigate the role of the IncRNA NEAT1 on the neuroepigenetic mechanisms of cognition.

\section{NEAT1 regulates the immediate early gene c-Fos involved in synaptic plasticity}

To investigate the role of NEAT1 at the transcriptomic level, we analyzed a publicly available RNA-seq dataset from iPSC-derived human neurons. Antisense oligo (ASO) knockdown of NEAT1 in $\mathrm{KCl}-$ treated human neurons revealed an extensive cohort of differentially expressed mRNAs. Knockdown alone was not sufficient to perturb the transcriptome in resting iPSC-derived human neurons, as evidenced by an imperfect separation via unsupervised hierarchical clustering prior to $\mathrm{KCl}$ stimulation (Fig. 2A). In contrast, NEAT1-knockdown appears to dramatically potentiate $\mathrm{KCl}$-driven differential expression of many genes (Fig. 2A-B).

To gain some insight into the health relevance for observed NEAT1-mediated changes in gene expression in human neurons, we queried the annotated disease classes from the Genetic Association Database via the Database for Annotation, Visualization and Integrated Discovery
(DAVID) ${ }^{25}$, and observed significant enrichment for three disease classes: cancer, renal, and aging. (Supplemental File 2). NEAT1-regulated genes appear to be non-randomly distributed among annotated biological processes (Fig. 2C), molecular functions (Supplemental File 2) and cellular components (Fig. 2D). Significant gene ontology (GO) term enrichment was partially consistent with previous observations of the NEAT1 regulatory axis, as we observed significant regulation of $\mathrm{GO}$ terms associated with viral gene expression; however, we also observed significant enrichment of GO terms important for hippocampal function, including the transcription factor AP-1 complex (GO:0035976, Fig. 2C-D).

The human Fos proto-oncogene (FOS, also known as C-FOS), a critical component of the AP-1 transcription factor subunit appeared to be overexpressed in human neurons after knocking down NEAT1 both in quiescent and $\mathrm{KCl}$-stimulated neurons and has a known relevance for hippocampus-dependent memory formation ${ }^{26}$ (Figs. $2 \mathrm{E}-\mathrm{F})$. Thus, we selected the murine homolog $c-F o s$ as a candidate gene for further studies of Neat1's regulatory potential.

\section{Neat1 is regulated by neuronal excitability and controls c-Fos gene expression}

As modeling Neat1 expression changes in response to in vivo neuronal activity and behavioral experience required the mammalian model organisms, we next sought to examine the regulatory capacity of Neat1 in rodent neurons. For this purpose, we knocked down murine Neat1 in the mouse Neuro-2a (N2a) cell line using small interfering RNAs (siRNAs). We observed that $24 \mathrm{~h}$ after treatment with Neat1-targeting siRNAs (Fig. $3 \mathrm{~A}$ ), expression of the c-Fos mRNA was significantly upregulated (Fig. 3B).

Interestingly, while our observations of c-Fos transcript expression in murine neurons recapitulated observations from human neurons, we observed that expression of the immediate early genes Egr1 and Btg2 were not overexpressed in mouse 


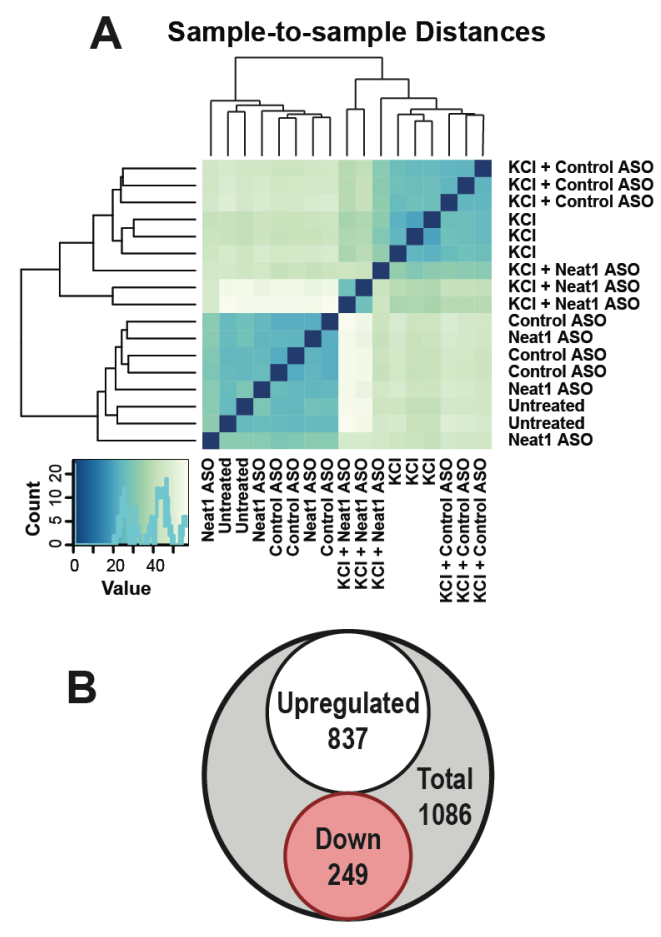

C GO analysis: Biological Processes

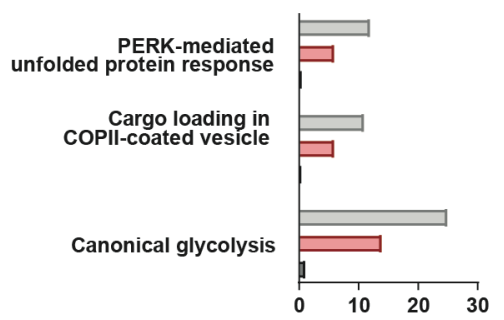

D Go analysis: Cellular components
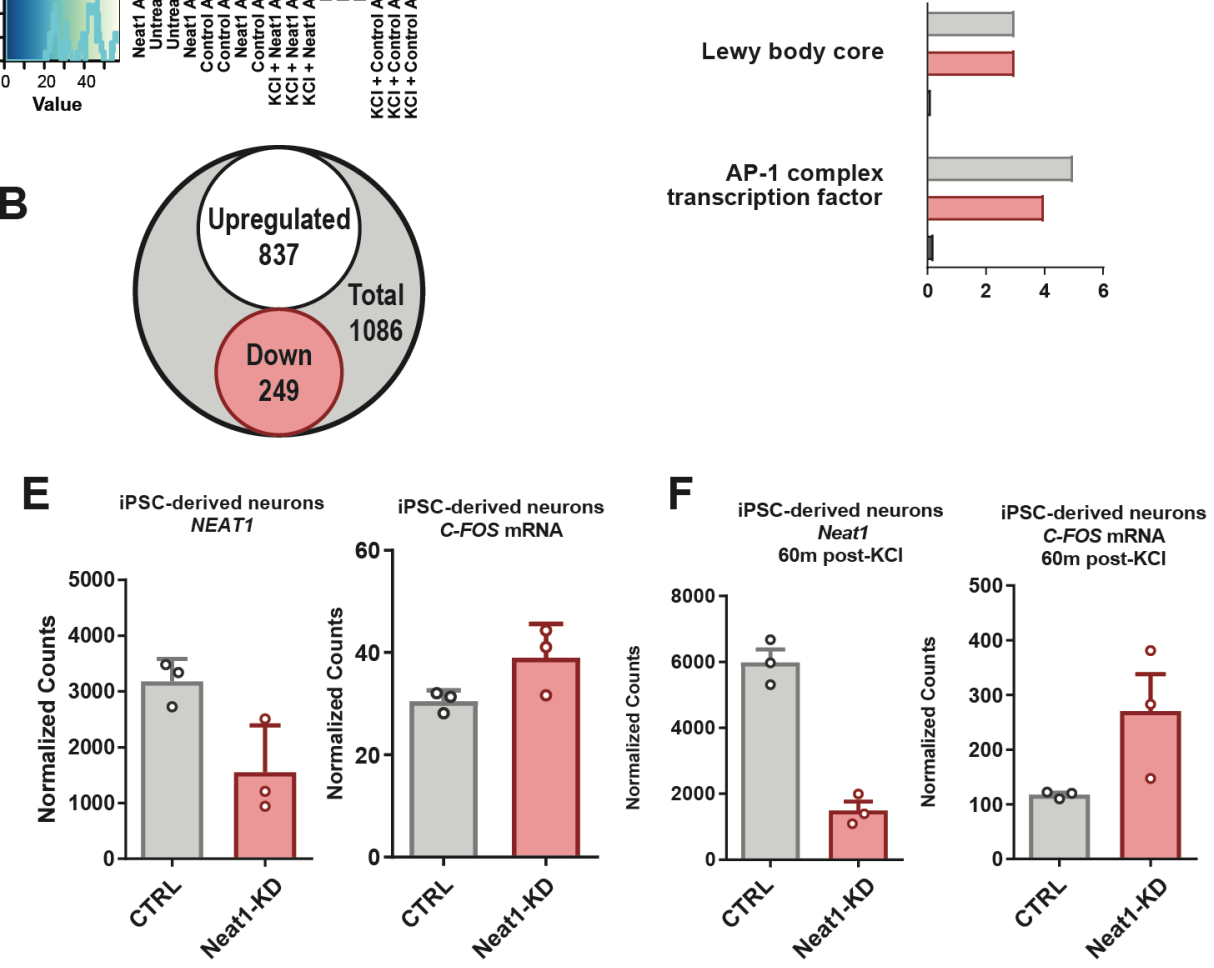

Figure 2. NEAT1 regulates expression of C-FOS mRNA and the AP-1 complex in iPSCderived human neurons.

(A) Unsupervised hierarchical clustering transcriptomes from Neat1 and $\mathrm{KCl}$ treated iPSCderived human neurons, based on DESeq2 normalized counts (B) Venn diagram depicting the total number of differentially expressed genes detected between $\mathrm{KCl}+$ Control_antisense oligonucleotide (ASO) and $\mathrm{KCl}+\mathrm{Neat1}$ _ASO groups via DESeq2. (C-D) Gene Ontology (GO) term enrichment for $D E$ genes depicted in panel B. All GO terms shown showed statistically significant enrichment $(\mathrm{BH}$ corrected $p<0.05)$ (E-F) Normalized count values for IncRNA NEAT1 and C-FOS mRNA either prior to $(\mathbf{E})$ or after (F) $\mathrm{KCl}$ treatment of iPSCderived neurons.
(Supplemental Fig. 2A-B) as they were in human neurons (Supplemental File 2), suggesting that there are species-specific regulatory differences in the Neat1 regulatory axis.

As mice with the c-Fos gene knocked out in the CNS show a specific loss of hippocampus-dependent spatial and associative learning tasks ${ }^{26}$, we next sought to investigate the relevance of Neat1 expression during memory consolidation after a hippocampus-dependent learning task. One hour after training in contextual fear conditioning we observed a significant reduction in the expression of Neat1 in the dorsal hippocampus coinciding with previously reported increases in expression of the $c$ Fos mRNA (Figs. 3C-D). As baseline expression of Neat1 in neurons is expected to be quite restricted compared to other cell types, we next stimulated neurons with $\mathrm{KCl}$ to ascertain the effect of activity on the expression of Neat1. Consistent with recent reports ${ }^{17}$, we observed that $\mathrm{KCl}$ stimulation drives a rapid reduction in Neat1 expression in both N2a cells (Supplementary Fig. 2E) and primary hippocampal pyramidal neurons, as recently reported (Figs. 3E-F), and consistent with the effects of context fear conditioning in vivo.

\section{Neat1 regulates $\mathrm{H} 3 \mathrm{~K} 9 \mathrm{me} 2 \mathrm{globally}$ and controls c- Fos promoter H3K9me2 and gene expression}

We next sought to investigate the c-Fos-relevant mechanisms of Neat1-orchestrated transcriptional control. To accomplish this, we used publicly available data assaying Neat1 chromatin binding via capture hybridization analysis of RNA targets and high-throughput sequencing (CHART-seq) in human MCF7 cells ${ }^{8}$. After mapping Neat1-bound peaks to the nearest transcription start sites, we observed that only a small subset of genes directly bound by Neat 1 are differentially expressed either after Neat1 knockdown or in the context of neuronal activation. However, we observed significant enrichment of Neat1 binding near genes associated with histone 
methyltransferase activity, including the $\mathrm{H} 3 \mathrm{~K} 9$ dimethyltransferase Ehmt1 (also known as GLP) (Figs. 4A-B; Supplemental File 3).

As c-Fos has previously been observed to be regulated by the Ehmt1/2 complex in the context of hippocampus-dependent memory formation ${ }^{27}$, we next sought to investigate the role of Neat1 in the regulation of histone methylation and $\mathrm{H} 3 \mathrm{~K} 9 \mathrm{me} 2$ specifically. After knockdown of Neat1 in neuronal cells, we observed that $\mathrm{H} 3 \mathrm{~K} 9 \mathrm{me} 2$ is reduced at a global scale while the expression of other histone modifications are unchanged (Fig. 4C-F). To ascertain whether the IncRNA Neat1 physically associates with the H3K9me2 methyltransferase complex in neurons, we performed RNA binding protein immunoprecipitations against the Ehmt2 subunit of the obligatory Ehmt1/2 heterodimer27-29. Consistent with recently published results ${ }^{6}$, we observed interaction between Neat1 and the H3K9me2 methyltransferase Ehmt2, suggesting multiple possible modes of action for Neat1-mediated regulation of $\mathrm{H} 3 \mathrm{~K} 9 \mathrm{me} 2$.
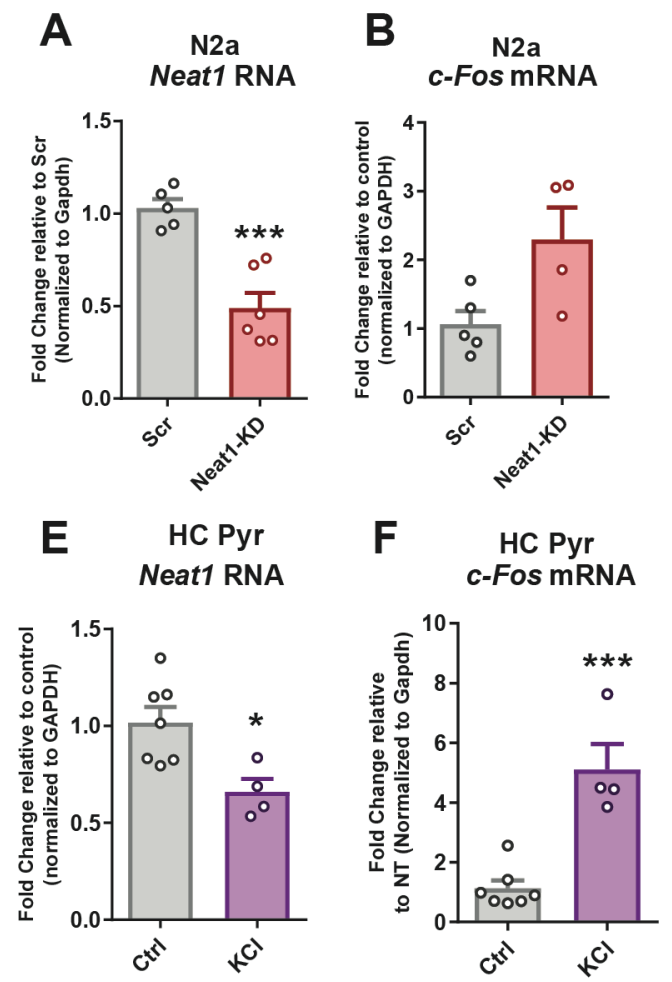

To assess the functional relevance of the Neat1H3K9me2 regulatory axis on the expression of $c$-Fos mRNA, we performed ChIP in conjunction with qPCR at the $c$-Fos promoter. We observed that after Neat1 knockdown with siRNAs, H3K9me2 at the c-Fos promoter was significantly depleted (Fig. 4G), consistent with observed changes in gene expression (Fig. 3B), while H3K9me2 within the c-Fos gene body were not significantly changed (Supplementary Fig. $2 \mathrm{~F})$.

\section{Neat1 knockdown regulates hippocampal memory formation and the epigenetic landscape at the c-Fos promoter in vivo}

Having demonstrated that Neat1 represses the epigenetic landscape and neuronal expression of the memory-critical c-Fos gene, we next sought to investigate the functional role of Neat1 expression on $c$-Fos promoter methylation and memory formation in vivo.

To ask whether Neat1 expression impacts hippocampus-dependent memory formation, we knocked down expression of Neat1 in hippocampal

C
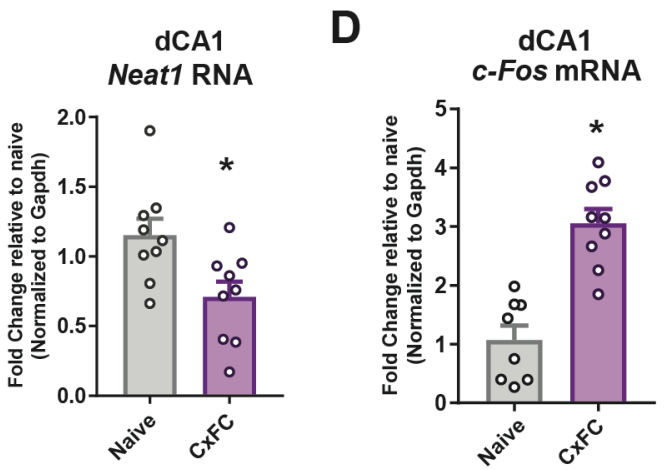

Figure 3. Neat1 regulates expression of $c$-Fos mRNA in murine neuronal cells (A) siRNA treated murine N2a cells show significantly reduced abundance of Neat1 transcript. $(n=5,6 ; p<0.0005)$ (B) Expression of c-Fos mRNA after treatment with Neat1-targeting siRNAs $(n=5,4 ; p=0.0335)$. (C-D) Neat1 expression is decreased (C; $n=9,9 ; p<0.0148)$ and $c$-Fos expression is increased ( $\mathbf{D} ; n=8,9 ; p<0.0001)$ in vivo in dorsal CA1 $1 \mathrm{~h}$ after training in contextual fear conditioning (E-F) Depolarization of rodent primary pyramidal neurons with $\mathrm{KCl}$ is sufficient to significantly reduce expression of Neat1 (E; $n=7,4 ; p=0.0147$ ) and reproduce commonly observed increases in $c$-Fos transcription $(\mathbf{F} ; n=7,4 ; p=0.0003)$. 
A

GO Enrichment: Molecular function

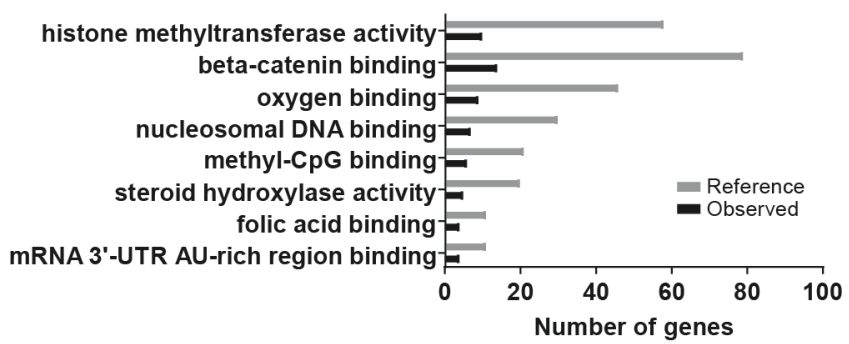

B

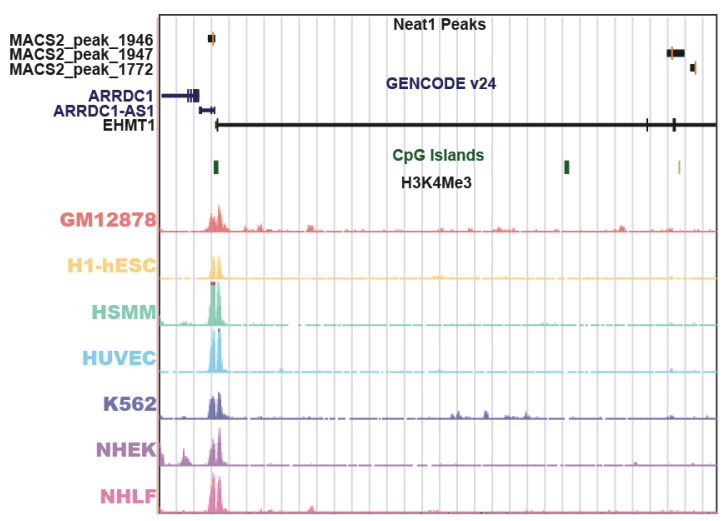

C

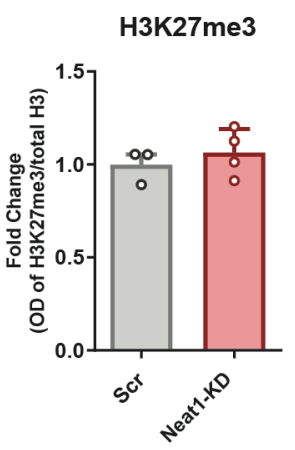

$\mathbf{F}$

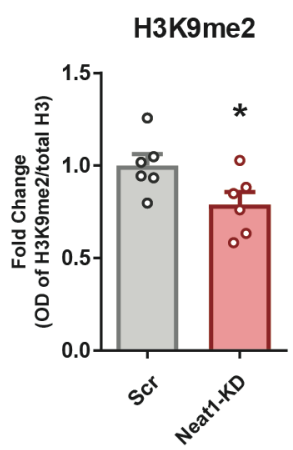

D

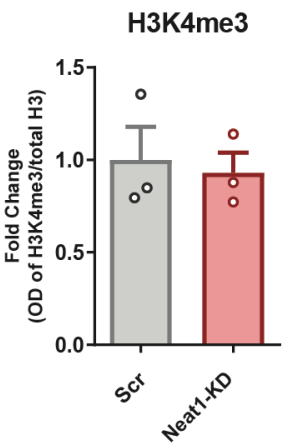

G

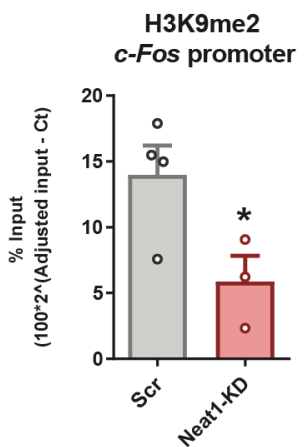

E

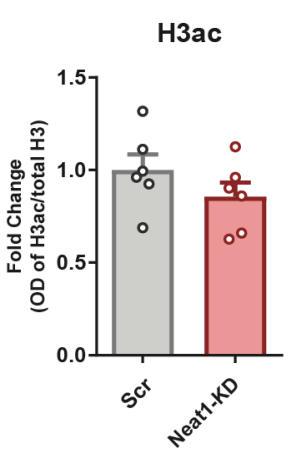

H

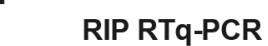
Neat1 RNA

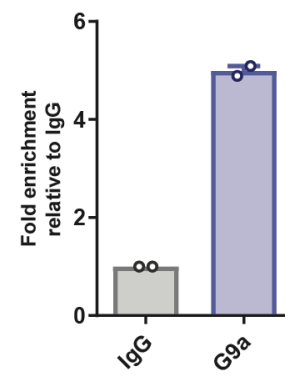

Figure 4. Neat1 modulates neuronal H3K9me2. (A) NEAT1 CHART-seq peaks were mapped to the nearest gene transcription start site, and functional enrichment was assessed using ChIPENRICH, with histone methyltransferase activity being noted as a significantly enriched $\mathrm{GO}$ term (BH corrected $p<0.05$ for all terms shown). (B) UCSC genome browser plot showing NEAT1binding peaks overlapping the human EHMT1 gene. (C-F) Graphs depicting changes in histone modifications in N2a cells after siRNA knockdown of Neat1 (C) H3K27me3 ( $\mathrm{n}=3$; $\mathrm{p}=0.4716)$ (D)H3K4me3 ( $n=3 ; p=0.7548)$ (E)H3ac $(n=6$; $\mathrm{p}=0.2377)(\mathbf{F}) \mathrm{H} 3 \mathrm{~K} 9 \mathrm{me} 2(n=6 ; p=0.0456)(\mathbf{G})$ ChIP-qPCR assay indicating a loss of H3K9me2 at the $c$-Fos gene promoter in N2a cells $(n=4,3$; $p=0.0472)(\mathrm{H})$ RNA binding protein immunoprecipitation for Ehmt2/Neat1 interaction $(n=2)$

(Supplemental Fig. 3A), mice had no differences in freezing behavior during the training phase of contextual fear conditioning, either before or after delivery of the foot shock (Fig. 5B). However, when returned to the training context $24 \mathrm{~h}$ later, mice that received Neat1-targeting siRNAs displayed significant increases in freezing behavior relative to mice that received nontargeting siRNAs (Fig. 5C).

To determine whether Neat1 expression impacts c-Fos promoter methylation in vivo, we sacrificed an additional cohort of behaviorally naïve animals five days after injection of siRNAs and performed ChIP-qPCR assays on one hemisphere of dorsal CA1 tissue collected from around the injection site. Consistent with our results in cultured neurons (Fig. 4G), we observed that concurrently with Neat1 knockdown five days after infusion with siRNAs, Neat1 knockdown significantly reduced $\mathrm{H} 3 \mathrm{~K} 9 \mathrm{me} 2$ at the c-Fos promoter in dorsal area CA1 (Fig. 5E). Thus, we hypothesized a model in which Neat1 expression might be regulating memory formation via epigenetic repression of $c$ Fos.

\section{Mimicking age-related upregulation of Neat1 is} sufficient to respectively restore or impair hippocampus-dependent memory formation

Numerous studies have reported overexpression

area CA1 by directly infusing Neat1-targeting siRNAs or non-targeted siRNAs and assayed long term memory function using contextual fear conditioning, a hippocampus-dependent memory task (Fig. 5A). We observed that five days after intra-CA1 injection of Neat1-targeting siRNAs, a time when we observe significant reduction in expression of Neat 1 of Neat 1 in senescing cells, as well as in aging CNS tissues in both humans and mice ${ }^{11,30}$. Upon comparing publicly available hippocampus RNA-seq datasets from 3 month-old young mice versus 24 month-old aged mice we observed upregulation of Neat1 relative to young animals, consistent with 

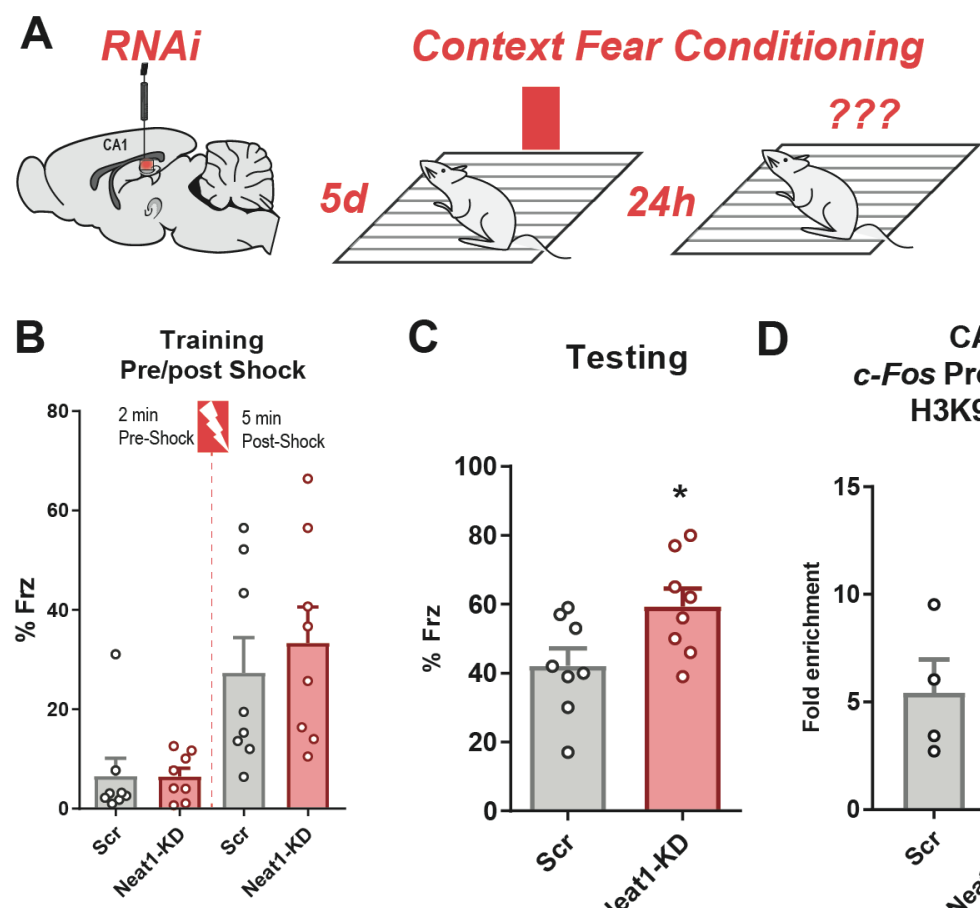

C

Testing

D c-Fos Promoter H3K9me2
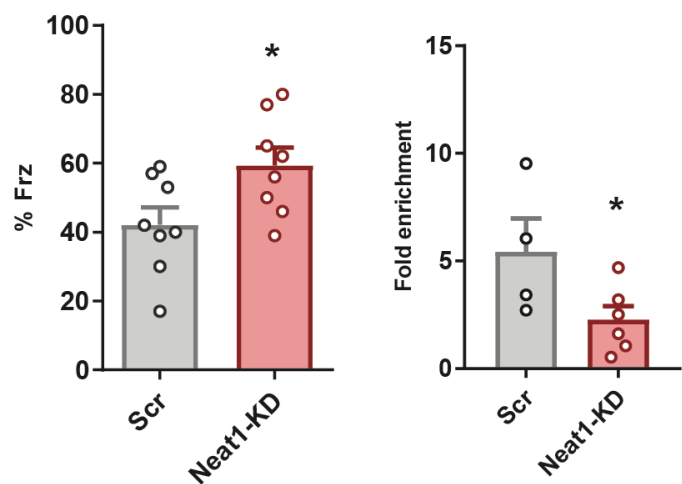

Figure 5. Neat1 knockdown regulates cFos promoter methylation in vivo and improves long-term memory (A) Graphic depiction of siRNA infusion into hippocampal area CA1 and single-pairing contextual fear conditioning paradigm. Briefly, male C57BL/6 mice were trained 5d after bilateral infusion of siRNAs and tested $24 \mathrm{~h}$ after training. (B) Freezing behavior as a percent of epoch during training phases of the contextual fear conditioning paradigm. No significant difference detected for either the Pre-shock $(n=8 ; p=0.9826)$ or Postshock ( $n=8 ; p=0.5626)$ epochs. (C) Freezing behavior as a percent of total time during the 5-min test trial $(n=8 ; p=$ 0.0307). (D) c-Fos promoter H3K9me2 remained depleted after Neat1-knockdown $5 d$ after the conclusion of behaviour experiments $(n=18,18 ; p=0.0450)$ previously reported results ${ }^{11}$ (Fig. 6A), as well as downregulation of c-Fos gene expression (Fig. 6B), consistent with previously reported age-associated hippocampus-dependent memory impairments ${ }^{11}$.

We next tested whether hippocampus-dependent memory formation might be improved in aged mice by knockdown of Neat1. To this end, we knocked down expression of Neat 1 in the hippocampal area CA1 of 18-19 month-old mice, an age at which we have previously observed significant upregulation of H3K9me2 in the aging rat hippocampus 31 (Supplemental Fig. 4), by directly infusing Neat1targeting siRNAs or non-targeted siRNAs and assayed long term memory function using contextual fear conditioning, with three pairings of the shock to the novel context (Fig. 6C). We observed that knockdown of Neat1 in the dorsal hippocampus of aged mice resulted in significant improvements in freezing after $24 \mathrm{~h}$ (Figs. 6D-E), but not during training, similar to results seen in young mice (Fig. $5 \mathrm{C})$.

We next sought to test the sufficiency of Neat1 overexpression to regulate performance in memory tasks, we designed a single guide RNA (sgRNA) targeting Neat1 for overexpression from the endogenous locus (Fig. 7A, Supplemental Fig. 3B-C), and delivered the CRISPRa system in vivo into dorsal CA1 via in vivo transfection (Fig. 7B). Mice were then trained in contextual fear conditioning with three pairings of the shock to the novel context (Fig. 6D). Animals overexpressing Neat1 from the endogenous locus (Neat1-OE) had no significant differences in freezing during the training period, either before or after exposure to the unconditioned stimulus (Fig. $6 \mathrm{E}$ ); however, when returned to the training context $24 \mathrm{~h}$ after training, Neat1-OE animals froze significantly less than control animals which received only the sgRNA plasmid (Fig. 6F), suggesting that elevated Neat1 in area CA1 is sufficient to impair hippocampus-dependent memory formation.

\section{DISCUSSION}

While previous studies have observed regulatory roles for the IncRNA NEAT1, including that NEAT1 localizes to chromatin and governs chromatin modification 8,9 , little work has been done to resolve this regulatory role of NEAT1 in the context of longterm memory formation. RNA sequencing analysis revealed that the human IncRNA NEAT1 binds to the EHMT1 locus and that NEAT1 knockdown regulates neuronal EHMT1 expression (Supplemental Data S1). We observed that murine Neat1 acts as a potent regulator of $\mathrm{H} 3 \mathrm{~K} 9 \mathrm{me} 2$ both in cultured cells and in vivo (see Fig. 4). Due to recent observations that NEAT1 interacts directly with Ehmt ${ }^{6}$, an observation which we ourselves have reproduced via RIP (see Fig. 4), we cannot yet ascertain whether transcriptional control of EHMT1 or direct interaction with the repressor complex is the rate-limiting factor for H3K9me2 abundance. This intricate multipoint interaction is perhaps illustrative of the intricate systems of regulatory feedback which are thought to control epigenetic mechanisms. Nonetheless, knockdown of Neat1 was sufficient to perturb this 
system and to result in both bulk and site-specific changes in $\mathrm{H} 3 \mathrm{~K} 9 \mathrm{me} 2$ in neurons.

Previous investigations as to the epigenetic regulatory role of Neat 1 have resulted in paradoxical observations to the effect that Neat1 binds to genomic loci and mediates activation of transcription ${ }^{9}$, but that suppression of Neat1 expression results primarily in increased neuronal gene expression ${ }^{17}$. We show here that Neat 1 induces widespread regulation of neuronal $\mathrm{H} 3 \mathrm{~K} 9 \mathrm{me} 2$, potentially resolving this dilemma and further explaining age-related increases in H3K9me2 previously observed in the hippocampus. Moreover, we observed that Neat1 expression is correlated with $\mathrm{H} 3 \mathrm{~K} 9 \mathrm{me} 2$ globally as well as at the promoter of the aging-repressed memory-related gene c-Fos. While Neat1 has been observed to act on and via numerous epigenetic mechanisms, to our knowledge this is the first observation that Neat1-mediated epigenetic mechanisms are sufficient to govern cognitive function.

Studies of the neuronal impact of Neat1 expression have thus far been limited to the context of neurological disorders, and in many cases to cultured neuronal cells. Our observations suggest that Neat1 plays a regulatory role in neuronal $\mathrm{H} 3 \mathrm{~K} 9 \mathrm{me} 2$ both in cultured neurons and in vivo, and that increases in Neat1 might play a significant role in the agerelated decline of hippocampus-dependent memory formation. In humans, expression of NEAT1 is generally limited in the CNS, and overexpression is a common hallmark of several neurological disorders. While experimental reduction of Neat1 has very recently been shown to have therapeutic potential in the context of such disorders, the impact of age-related changes in expression has remained unexplored until now. Interestingly, while our experiments were designed to investigate the age-related impact of Neat1, we note that recent experiments have described neuroinflammation-mediated increases in Neat1 expression ${ }^{32}$, and that the findings described in this manuscript implicate Neat1 as a potential mechanism by which neuroinflammation might impact memory.

While the experiments described here are largely sufficient to explain prior observations of elevated H3K9me2 in the aging hippocampus (Supplementary Fig. 4;31, our experiments indicate that increased expression of Neat 1 is not sufficient to explain all of the aging-related neuroepigenetic changes observed in this region. It is likely that many hippocampal IncRNAs have distinct or overlapping roles in the regulation of the neuroepigenetic aging process. Indeed, human NEAT1 itself has been observed to associate with multiple chromatin modifying enzymes 6,33-35. Although we did not detect significant regulation of histone modifications other than H3K9me2 at the global level after knockdown of Neat1, the absence of such observations does not preclude the existence of biologically or behaviorally meaningful epigenetic
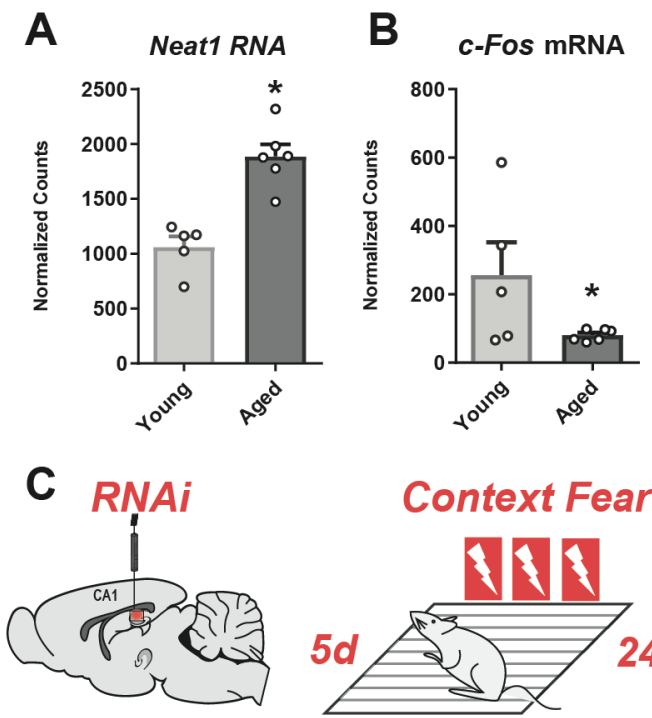

Context Fear Conditioning
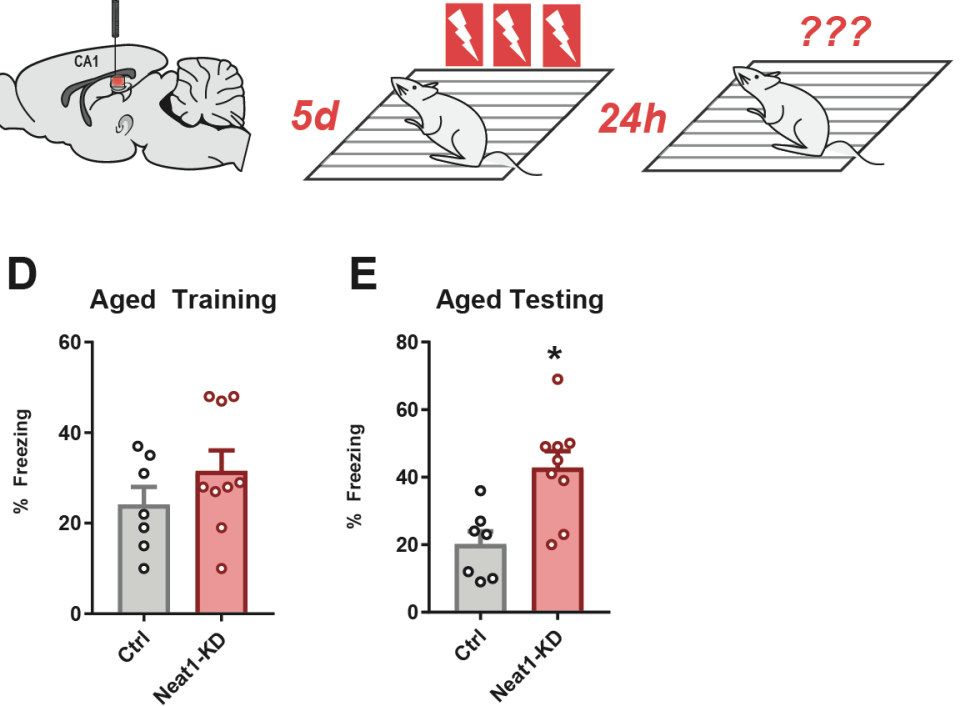

Figure 6. Neat1-knockdown improves long-term memory in aged animals (A-B) DESeq2-generated normalized counts of RNAseq data from $3 \mathrm{mo}$ and $24 \mathrm{mo}$ C57/B6 mice. Neat1 expression (A) was significantly elevated and c-Fos mRNA (B) was significantly repressed in aged hippocampi relative to the hippocampi of young mice. (C) Graphic depiction of siRNA infusion into hippocampal area CA1 and single-pairing contextual fear conditioning paradigm. Briefly, 18 mo old male C57/ B6 mice were trained $5 \mathrm{~d}$ after bilateral infusion of siRNAs and tested 24h after training. (D-E). Aged mice (18 month-old) were trained with three pairings of shock with a novel context after knockdown of Neat1 and demonstrated no significant difference during training (D) $(n=7,9 ; p=0.2496)$, but significantly enhanced freezing $24 \mathrm{~h}$ after testing $(\mathrm{E})(n=7,9 ; p=0.0039)$. 

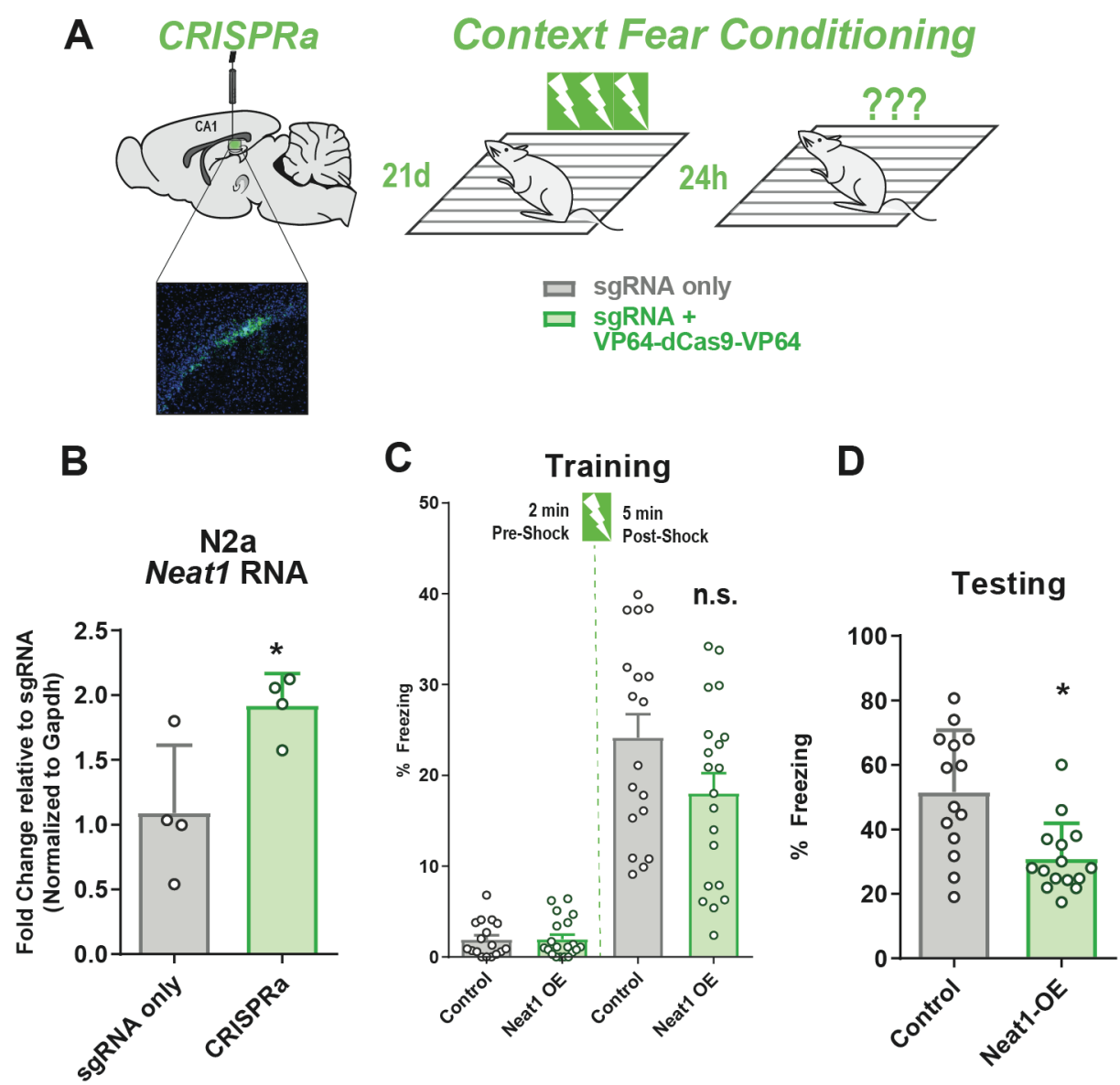

Figure 7. Mimicking age-related Neat1 overexpression via CRISPRa impairs hippocampal memory formation (A) Graphic depiction CRISPRa system infusion into hippocampal area CA1, with visualization of hippocampal expression of EGFP fluorescent marker, and three-pairing contextual fear conditioning paradigm. Briefly, male C57BL/6 mice (3-7 month-old) were trained 21d after bilateral infusion of either sgRNA plasmid alone or co-delivered with a transcription-activating dCas9-effector protein and tested $24 \mathrm{~h}$ after training. (B) Confirmation of efficacy of CRISPRa system to upregulate Neat1 expression in murine neurons (C). Freezing behavior as a percent of epoch during training phases of the contextual fear conditioning paradigm. No significant difference detected for either the Pre-shock $(n=18 ; p=0.3476)$ or Post-first shock epochs $(n=18 ; p=0.0665)$. (D) Freezing behavior as a percent of total time during the 5 min test trial $(n=18 ; p=0.0450)$.

regulation that is more limited in scope and might be uncovered in future studies with a large-scale sequencing approach.

In this work, we demonstrate that Neat1 regulates a critical transcriptional pathway for hippocampusdependent memory in rodent neurons in vitro, in vivo, and likewise in iPSC-derived human neurons. While attempts to establish the functionality of the evolutionarily conserved IncRNA Neat1 have met with limited success, little has yet been done to functionally characterize Neat1 in the context of cognition. Here, we observed that the IncRNA Neat1 may serve as an endogenous molecular brake on the formation of hippocampus-dependent spatial memories.

\section{Acknowledgements}

This work was supported by grants from the National Institute of Mental Health (FDL: MH082106, MH097909) and the Evelyn F. McKnight Brain Research Foundation.

\section{Declaration of Interest}

The authors declare no competing financial interests. 


\section{REFERENCES}

1. Hutchinson, J. N. et al. A screen for nuclear transcripts identifies two linked noncoding RNAs associated with SC35 splicing domains. BMC Genomics 8, 39 (2007).

2. Clemson, C. M. et al. An architectural role for a nuclear noncoding RNA: NEAT1 RNA is essential for the structure of paraspeckles. Mol Cell 33, 717-726 (2009).

3. Yamazaki, T. et al. Functional Domains of NEAT1 Architectural IncRNA Induce Paraspeckle Assembly through Phase Separation. Mol Cell 70, 1038-1053.e7 (2018).

4. Li, R., Harvey, A. R., Hodgetts, S. I. \& Fox, A. H. Functional dissection of NEAT1 using genome editing reveals substantial localization of the NEAT1_1 isoform outside paraspeckles. RNA 23, 872-881 (2017).

5. Imamura, K. et al. Long noncoding RNA NEAT1dependent SFPQ relocation from promoter region to paraspeckle mediates IL8 expression upon immune stimuli. Mol Cell 53, 393-406 (2014).

6. Li, Y. \& Cheng, C. Long noncoding RNA NEAT1 promotes the metastasis of osteosarcoma via interaction with the G9a-DNMT1-Snail complex. Am J Cancer Res 8, 81-90 (2018).

7. Chen, Q. et al. Long Noncoding RNA NEAT1, Regulated by the EGFR Pathway, Contributes to Glioblastoma Progression Through the WNT/ $\beta$ Catenin Pathway by Scaffolding EZH2. Clin Cancer Res 24, 684-695 (2018).

8. West, J. A. et al. The long noncoding RNAs NEAT1 and MALAT1 bind active chromatin sites. Mol Cell 55, 791-802 (2014).

9. Chakravarty, D. et al. The oestrogen receptor alpha-regulated IncRNA NEAT1 is a critical modulator of prostate cancer. Nat Commun 5, 5383 (2014).

10. Yu, X., Li, Z., Zheng, H., Chan, M. T. V. \& Wu, W. K. K. NEAT1: A novel cancer-related long noncoding RNA. Cell Prolif 50, (2017).

11. Stilling, R. M. et al. De-regulation of gene expression and alternative splicing affects distinct cellular pathways in the aging hippocampus. Front Cell Neurosci 8, 373 (2014).

12. Li, J. et al. Relationship between schizophrenia and changes in the expression of the long noncoding RNAs Meg3, Miat, Neat1 and Neat2. J Psychiatr Res 106, 22-30 (2018).

13. Sunwoo, J.-S. et al. Altered expression of the long noncoding RNA NEAT1 in huntington's disease. Mol Neurobiol 54, 1577-1586 (2017).
14. Yan, W., Chen, Z.-Y., Chen, J.-Q. \& Chen, H.-M. LncRNA NEAT1 promotes autophagy in MPTPinduced Parkinson's disease through stabilizing PINK1 protein. Biochem Biophys Res Commun 496, 1019-1024 (2018).

15. Liu, Y. \& Lu, Z. Long non-coding RNA NEAT1 mediates the toxic of Parkinson's disease induced by MPTP/MPP+ via regulation of gene expression. Clin Exp Pharmacol Physiol 45, 841848 (2018).

16. Puthiyedth, N., Riveros, C., Berretta, R. \& Moscato, P. Identification of Differentially Expressed Genes through Integrated Study of Alzheimer's Disease Affected Brain Regions. PLoS ONE 11, e0152342 (2016).

17. Barry, G. et al. The long non-coding RNA NEAT1 is responsive to neuronal activity and is associated with hyperexcitability states. Sci Rep 7, 40127 (2017).

18. Lipovich, L. et al. Activity-dependent human brain coding/noncoding gene regulatory networks. Genetics 192, 1133-1148 (2012).

19. ENCODE Project Consortium. An integrated encyclopedia of DNA elements in the human genome. Nature 489, 57-74 (2012).

20. Carithers, L. J. et al. A Novel Approach to HighQuality Postmortem Tissue Procurement: The GTEx Project. Biopreservation and biobanking 13, 311-319 (2015).

21. GTEx Consortium. The Genotype-Tissue Expression (GTEx) project. Nat Genet 45, 580585 (2013).

22. Darmanis, S. et al. Single-Cell RNA-Seq Analysis of Infiltrating Neoplastic Cells at the Migrating Front of Human Glioblastoma. Cell Rep 21, 1399-1410 (2017).

23. Barry, G., Guennewig, B., Fung, S., Kaczorowski, D. \& Weickert, C. S. Long Non-Coding RNA Expression during Aging in the Human Subependymal Zone. Front Neurol 6, 45 (2015).

24. Pereira Fernandes, D., Bitar, M., Jacobs, F. M. J. \& Barry, G. Long Non-Coding RNAs in Neuronal Aging. Non-coding RNA 4, (2018).

25. Huang, D. W., Sherman, B. T. \& Lempicki, R. A. Systematic and integrative analysis of large gene lists using DAVID bioinformatics resources. Nat Protoc 4, 44-57 (2009).

26. Fleischmann, A. et al. Impaired long-term memory and NR2A-type NMDA receptordependent synaptic plasticity in mice lacking cFos in the CNS. J Neurosci 23, 9116-9122 (2003).

27. Gupta-Agarwal, S. et al. G9a/GLP histone lysine dimethyltransferase complex activity in the hippocampus and the entorhinal cortex is required for gene activation and silencing during 
memory consolidation. J Neurosci 32, 54405453 (2012).

28. Sharma, M., Razali, N. B. \& Sajikumar, S. Inhibition of G9a/GLP Complex Promotes LongTerm Potentiation and Synaptic Tagging/Capture in Hippocampal CA1 Pyramidal Neurons. Cereb Cortex 27, 3161-3171 (2017).

29. Tachibana, M., Matsumura, Y., Fukuda, M., Kimura, H. \& Shinkai, Y. G9a/GLP complexes independently mediate H3K9 and DNA methylation to silence transcription. EMBO J 27, 2681-2690 (2008).

30. Akbarian, S. Epigenetics of schizophrenia. Current topics in behavioral neurosciences 4, 611-628 (2010).

31. Morse, S. J., Butler, A. A., Davis, R. L., Soller, I. J. \& Lubin, F. D. Environmental enrichment reverses histone methylation changes in the aged hippocampus and restores age-related memory deficits. Biology 4, 298-313 (2015).

32. Li, Z. et al. Emerging roles of long non-coding RNAs in neuropathic pain. Cell Prolif e12528 (2018). doi:10.1111/cpr.12528

33. Hirose, T. et al. NEAT1 long noncoding RNA regulates transcription via protein sequestration within subnuclear bodies. Mol Biol Cell 25, 169183 (2014).

34. Murthy, U. M. S. \& Rangarajan, P. N. Identification of protein interaction regions of VINC/NEAT1/Men epsilon RNA. FEBS Lett 584, 1531-1535 (2010).

35. Spiniello, M. et al. HyPR-MS for multiplexed discovery of MALAT1, NEAT1, and NORAD IncRNA protein interactomes. J Proteome Res (2018). doi:10.1021/acs.jproteome.8b00189

36. Meadows, J. P. et al. DNA methylation regulates neuronal glutamatergic synaptic scaling. Sci Signal 8, ra61 (2015).

37. Jarome, T. J., Butler, A. A., Nichols, J. N., Pacheco, N. L. \& Lubin, F. D. NF-KB mediates Gadd $45 \beta$ expression and DNA demethylation in the hippocampus during fear memory formation. Front Mol Neurosci 8, 54 (2015).

38. Rinn, J. L. et al. Functional demarcation of active and silent chromatin domains in human HOX loci by noncoding RNAs. Cell 129, 1311-1323 (2007).

39. Krueger, F. Trim Galore!: A wrapper tool around Cutadapt and FastQC to consistently apply quality and adapter trimming to FastQ files. (2015).

40. Kim, D., Langmead, B. \& Salzberg, S. L. HISAT: a fast spliced aligner with low memory requirements. Nat Methods 12, 357-360 (2015).

41. Love, M. I., Huber, W. \& Anders, S. Moderated estimation of fold change and' ' dispersion for
RNA-seq data with DESeq2. Genome Biol 15, 550 (2014).

42. Ashburner, M. et al. Gene ontology: tool for the unification of biology. The Gene Ontology Consortium. Nat Genet 25, 25-29 (2000).

43. The Gene Ontology Consortium. Expansion of the Gene Ontology knowledgebase and resources. Nucleic Acids Res 45, D331-D338 (2017).

44. Chalei, V. et al. The long non-coding RNA Dali is an epigenetic regulator of neural differentiation. elife 3, e04530 (2014).

45. Feng, J., Liu, T., Qin, B., Zhang, Y. \& Liu, X. S. Identifying ChIP-seq enrichment using MACS. Nat Protoc 7, 1728-1740 (2012).

46. Welch, R. P. et al. ChIP-Enrich: gene set enrichment testing for ChIP-seq data. Nucleic Acids Res 42, e105 (2014). 


\section{METHODS}

Animal housing. Naïve 3-7 month-old or 18 month-old C57BL/6 mice were group housed (2-7 animals/cage) in plastic cages with ad libitum access to food and water and were maintained on a 12-h light/dark cycle. All behavioral tests were conducted during the light cycle, and all procedures were approved by the University of Alabama at Birmingham Institutional Animal Care and Use Committee and done in accordance with the National Institute of Health ethical guidelines.

Cell culture. N2A cells were maintained in DMEM supplemented with $10 \%$ FBS. After thawing, the cells were passaged a minimum of two times prior to use in experiments. The cells were kept at $37^{\circ} \mathrm{C}$ in a $5 \% \mathrm{CO}_{2}$ incubator. Dissociated cultures of hippocampal pyramidal cells were obtained from embryonic day 18 rat embryos as described previously ${ }^{36}$. Briefly, timed-pregnancy female Sprague-Dawley rats were terminally anesthetized and embryos were removed from the uterus, then transferred to Hank's balanced salt solution (HBSS, Gibco) for dissection. Primary rat hippocampal neurons were dissociated via incubation with papain for 20 min at $37^{\circ} \mathrm{C}$, rinsed in HBSS, then resuspended in Neurobasal medium (Gibco) and further mechanically dissociated by passing through a series of progressively smaller fire-polished glass Pasteur pipettes. The

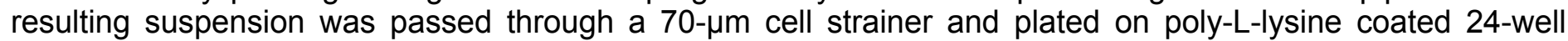
plates ( $7.5 \times 10^{4}$ cells per well). Cells were maintained for 2 weeks in Neurobasal supplemented with B-27 and Glutamax (Thermo Fisher Scientific) at $37^{\circ} \mathrm{C}$ and $5 \% \mathrm{CO}_{2}$. For $\mathrm{KCl}$ stimulation, $6.25 \mu \mathrm{L} 1 \mathrm{M} \mathrm{KCl}$ (Sigma) was added to two-week in vitro cultures, for a final concentration of $12.5 \mathrm{mM} \mathrm{KCl}$.

siRNA Delivery. Young, 3-7 month-old mice were anesthetized with an intraperitoneal injection of ketaminedexmedetomidine and received bilateral intra-CA1 injections of Lincode SMARTpool siRNAs (Dharmacon) targeting the murine Neat1 (\#R-160022-00-0005) or a negative control (\#D-001320-10-05), conjugated with in vivo JetPEI (PolyPlus Transfection), an in vivo transfection reagent, at the stereotaxic coordinates (AP $-2.0 \mathrm{~mm}$, $\mathrm{ML} \pm 1.5 \mathrm{~mm}, \mathrm{DV}-1.7 \mathrm{~mm})$ with respect to bregma. Aliquots of siRNA stocks $(100 \mu \mathrm{M})$ were diluted to a concentration of $\sim 2.5 \mu \mathrm{M}$ and conjugated with in vivo JetPEI on the day of surgery. Infusions were given over a $10 \mathrm{~min}$ period $(0.1 \mu \mathrm{L}$ per $\mathrm{min})$ for a total volume of $1 \mu \mathrm{L}$ per hemisphere. After a $48 \mathrm{~h}$ recovery period, mice were handled daily for $>3 \mathrm{~min}$ and trained in contextual fear conditioning at five days post-surgery. Aged (18-19 month old) mice were treated similarly but were anesthetized with vaporized isoflurane $(3 \%$ induction, $2 \%$ maintenance). Mice were sacrificed at ten days post-surgery and dorsal area CA1 was harvested from each hemisphere.

CRISPRa Delivery. Mice were anesthetized with an intraperitoneal injection of ketamine-dexmedetomidine and received bilateral intra-CA1 injections of a guide RNA expression vector driven by the murine U6 promoter and targeting the murine Neat1 promoter region (Addgene \#44248) either alone or in conjunction with an expression vector coding for the S. pyogenes dCas9 fused to two copies of the VP64 transactivator domain (Addgene \#59791). Endotoxin-free plasmids were purified using an endotoxin-free plasmid DNA purification kit (Machery-Nagel) and aliquoted to minimize freeze-thaw cycles. Endotoxin-free plasmid stocks were diluted to a final concentration of $\sim 500 \mathrm{ng} / \mathrm{uL}$ in sterile $10 \%$ glucose and incubated with in vivo JetPEI for 15 min at room temperature on the day of surgery. The resulting transfection complex was delivered via direct infusion at the stereotaxic coordinates (AP $-2.0 \mathrm{~mm}, \mathrm{ML} \pm 1.5 \mathrm{~mm}, \mathrm{DV}-1.4 \mathrm{~mm}$ ) with respect to bregma. Infusions were given over a $10 \mathrm{~min}$ period $(0.1 \mu \mathrm{L}$ per $\mathrm{min})$ for a total volume of $1 \mu \mathrm{L}(\sim 500 \mathrm{ng}$ plasmid DNA) per hemisphere.

Contextual fear conditioning. Mice were trained to either a weak or strong contextual fear conditioning (CFC) paradigm in a novel context, and long term memory was assessed upon returning the animals to the training context $24 \mathrm{~h}$ after training. The weak CFC paradigm consisted of a $118 \mathrm{~s}$ baseline followed by a single shock $(0.5 \mathrm{~mA}, 2 \mathrm{sec})$ pairing in the novel context, while the strong CFC paradigm consisted of a 119-sec baseline followed by three shock pairings $(0.5 \mathrm{~mA}, 1 \mathrm{~s})$ with interleaved rest periods of $59 \mathrm{sec}$ each. Twenty-four $\mathrm{h}$ after training, animals were placed back into the training context for five min to test retention. Freezing behavior was scored by Med Associates software.

Collection of whole area CA1. One hour after training, the whole brain was removed by gross dissection and placed in oxygenated $\left(95 \% / 5 \% \mathrm{O}_{2} / \mathrm{CO}_{2}\right)$ ice-cold cutting solution $(110 \mathrm{mM}$ sucrose, $60 \mathrm{mM} \mathrm{NaCl}, 3 \mathrm{mM} \mathrm{KCl}$, $1.25 \mathrm{mM} \mathrm{NaH}_{2} \mathrm{PO}_{4}, 28 \mathrm{mM} \mathrm{NaHCO}_{3}, 0.5 \mathrm{mM} \mathrm{CaCl}_{2}, 7 \mathrm{mM} \mathrm{MgCl}_{2}, 5 \mathrm{mM}$ glucose, and $0.6 \mathrm{mM}$ ascorbate). The CA1 region of the hippocampus was then microdissected from each hemisphere and flash frozen on dry ice. 
Collection of dorsal area CA1. Animals were sacrificed by cervical dislocation after overdosing with isoflurane at experiment-specific time points, and the whole brain was rapidly removed and immediately frozen on dry ice. The CA1 region of the dorsal hippocampus was then dissected out with the aid of a mouse brain matrix (Harvard Apparatus) to collect the area of CA1 targeted by siRNA or CRISPRa infusions. All tissue was stored at $-80^{\circ} \mathrm{C}$ prior to processing.

Western blotting. Normalized proteins $(2-10 \mu \mathrm{g})$ were separated via electrophoresis on either $10 \%$ or $20 \%$ polyacrylamide gels, transferred onto an Immobilon-FL membrane using a turbo transfer system (Biorad). Membranes were blocked in Licor blocking buffer and probed with the following primary antibodies for histone H3 (1:1000; Abcam \#ab1791), H3K9me2 (1:1000; Millipore \#07-441), H3K27me3 (1:1000; Millipore \#07-449), H3K4me3 (1:1000; Millipore \#04-745). Secondary goat anti-rabbit 700CW antibody (1:20,000; Licor Biosciences) was used for detection of proteins using the Licor Odyssey system. All protein quantification was done using ImageStudio Lite software (Licor).

Reverse transcription qPCR (RT-qPCR). Section text RNA was extracted from isolated CA1 or cultured cells using Trizol reagent according to the manufacturer's recommended protocol (Fisher). RNA yield was quantified spectrophotometrically (Nanodrop 2000c), and 200ng of RNA was DNAse treated (Amplification grade DNAse I, Sigma), converted to cDNA (iScript cDNA synthesis kit; Biorad), and PCR amplified on the CFX1000 real-time PCR system (BioRad), with primer annealing temperatures of $60^{\circ} \mathrm{C}$. See supplemental table for full descriptions of primers used. All data were analyzed using the delta delta Ct method.

Cell culture. ChIP was performed as described previously 31,37 . Briefly, samples were fixed in PBS with $1 \%$ formaldehyde for ten minutes at room temperature, chromatin was sheared using a Bioruptor XL on high power, lysates cleared by centrifugation and diluted in TE and RIPA buffer. Extracts were mixed with MagnaChIP protein A/G beads and immunoprecipitations were carried out at $4^{\circ} \mathrm{C}$ overnight with $5 \mu \mathrm{g}$ primary antibody (anti-H, Abcam \#ab40542; anti-Ezh2, \#ab3748) or no antibody (control). Immune complexes were sequentially washed with low salt buffer $(20 \mathrm{mM}$ Tris, $\mathrm{pH}$ 8.0, 0.1\% SDS, 1\% Triton X-100, 2 mM EDTA, 150 $\mathrm{mM} \mathrm{NaCl}$ ), high salt buffer (20 mM Tris, pH 8.1, 0.1\% SDS, 1\% Triton X-100, $500 \mathrm{mM} \mathrm{NaCl,} 1 \mathrm{mM}$ EDTA), LiCl immune complex buffer $(0.25 \mathrm{M} \mathrm{LiCl}, 10 \mathrm{mM}$ Tris, $\mathrm{pH}$ 8.1, 1\% deoxycholic acid, 1\% IGEPAL-CA630, $500 \mathrm{mM}$ $\mathrm{NaCl}, 2 \mathrm{mM}$ EDTA), and TE buffer, and eluted into 1xTE containing 1\% SDS. Protein-DNA crosslinks were reversed by heating at $65^{\circ} \mathrm{C}$ overnight. After proteinase $\mathrm{K}$ digestion $\left(100 \mu \mathrm{g} ; 2 \mathrm{~h}\right.$ at $\left.37^{\circ} \mathrm{C}\right)$, DNA was purified by phenol/chloroform/isoamyl alcohol extraction and ethanol precipitation. Immunoprecipitated DNA was quantified via spectrophotometry (Nanodrop 2000c) and $15 \mathrm{ng}$ of DNA from each sample was assayed via quantitative real-time PCR using primers specific to mouse genes of interest. See supplemental table for full descriptions of primers used.

RNA binding protein immunoprecipitation (RIP). RIP was performed as described previously 38 . Briefly, 5ug of primary antibody against Ehmt2 (Abcam \#ab40542), Ezh2(Abcam \#ab3748) or normal rabbit IgG (Cell signaling) were conjugated with 25uL MagnaChIP protein A/G beads (EMD Millipore). Freshly harvested nuclear pellets from at least $10^{\wedge} 6$ N2a cells were sheared via Dounce homogenization (15-20 strokes) in RIP buffer (150 mM KCl, $25 \mathrm{mM}$ Tris pH 7.4, 5 mM EDTA, $0.5 \mathrm{mM}$ DTT, 0.5\% NP40, 1x Protease Inhibitor Cocktail (Sigma), $100 \mathrm{U} / \mathrm{ml}$ SUPERASin (Ambion), cleared via centrifugation at 13,000 RPM to remove nuclear membrane and debris, and split into fractions for IP. Sheared nuclear extracts were mixed with antibody conjugated MagnaChIP protein A/G beads and immunoprecipitations were carried out at $4^{\circ} \mathrm{C}$ for four hours. Beads were then immobilized on a magnetic tube rack, and immune complexes were sequentially washed three times with RIP buffer. Beads were then resuspended in $1 \mathrm{~mL}$ of Trizol (Thermo Fisher), and coprecipitated RNAs were isolated according to the manufacturer's recommended protocol. RT-qPCR for Neat1 was then performed as described above.

Statistical analyses. Data from all experiments were analyzed using Analysis of Variance (ANOVA) with Fisher LSD post hoc test or with Student's t-test unless otherwise noted in the figure legend. Values reported in the text and error bars are the mean \pm SEM unless otherwise noted. All datasets were screened for outliers prior to analysis via Grubb's test $(a=0.05)$ and outliers were subsequently excluded. Statistical tests were performed in R or Prism 7 (GraphPad). Nonparametric tests were used where appropriate and tests were 2tailed unless otherwise noted. For all experiments, $n$ indicates the number of biological replicates. For cell 
culture experiments, this indicates the number of independently growing flasks or wells. For experiments involving animal behavior, this indicates the number of animals used. For experiments involving tissue collection from animals, this indicates the number of animals we collected the tissue from.

GTEx data. Data from the GTEx Analysis Release V7 (dbGaP Accession phs000424.v7.p2) were obtained via the GTEx portal web tool. Expression values plotted are in transcripts per million (TPM), using the GENCODE annotated transcript for isoforms or a gene level model based on the GENCODE model with isoforms collapsed to single genes. Isoform expression values were hierarchically clustered using Euclidean distance and average linkage; dendrogram scale shows cluster distance.

Analysis of bulk RNAseq and ChIPseq data. Single or paired-end RNAseq data was imported into the public Galaxy server at usegalaxy.org directly from the European Nucleotide Archive (study accession numbers PRJEB9006 and PRJNA262674) in FASTQ format and run through a standardized workflow consisting of quality trimming via Trim Galore! 39 (Galaxy Version 0.4.2), read alignment to via HISAT40 (Galaxy Version 2.0.3), and feature counting via featureCounts (Galaxy Version 1.4.6.p5). Individual count files were grouped by treatment (Animal age) and differential expression testing was performed using DESeq241 (Galaxy Version 2.11.39). All reference genomes and annotations were obtained from Gencode releases current at the time of analysis, including the Genome Reference Consortium Mouse Build 38 patch release 5 (GRCm38.p5) and evidence-based annotation of the mouse genome (GRCm38), version M16 (Ensembl 91), human build GRCh38 and the human annotation Release 25 (GRCh38.p7). Gene ontology (GO) enrichment was assessed using a PANTHER Overrepresentation Test web tool provided by the Gene Ontology Consortium 42,43 (release date 2017-11-28). DAVID functional annotation was used to assess gene set enrichment for GAD_DISEASE_CLASS using default settings (DAVID 6.8).

CHART-seq data was accessed via NIH SRA Toolkit from accession PRJNA252626 and analyzed using similar read quality control and alignment tools as described above. CHART-seq peaks were called using the MACS2 algorithm ${ }^{44,45}$. Overlapping peaks were combined into a single peak, as recommended for input into ChIP-Enrich package. Using the ChIP-Enrich R package ${ }^{46}$ (version 2.4.0), CHART-seq peaks from MACS2 were assigned to the nearest transcription start site and GO Enrichment was assessed for Biological Processes and Molecular Functions.

\section{scRNA-seq analysis}

Data were obtained via the European Bioinformatics Institute's Single-cell Expression Atlas. T-distributed Stochastic Neighbor Embedding (t-SNE) plots were constructed using transcript per million (TPM) values from the transcriptomes of 3,589 single cells biopsied from four glioblastoma patients ${ }^{22}$. Unbiased clusters were generated using a t-SNE perplexity of 10; plots were colored via biased inferred cell type, as reported by the authors of the dataset. Biopsied tissue included cells from the tumor core as well as peripheral tissue; however, all cells inferred to be neurons were collected from noncancerous tissue adjacent to the glioblastoma. 
bioRxiv preprint doi: https://doi.org/10.1101/531707; this version posted January 30, 2019. The copyright holder for this preprint (which was not certified by peer review) is the author/funder. All rights reserved. No reuse allowed without permission.

\section{SUPPLEMENTAL FIGURES}


bioRxiv preprint doi: https://doi.org/10.1101/531707; this version posted January 30, 2019. The copyright holder for this preprint (which was not certified by peer review) is the author/funder. All rights reserved. No reuse allowed without permission.
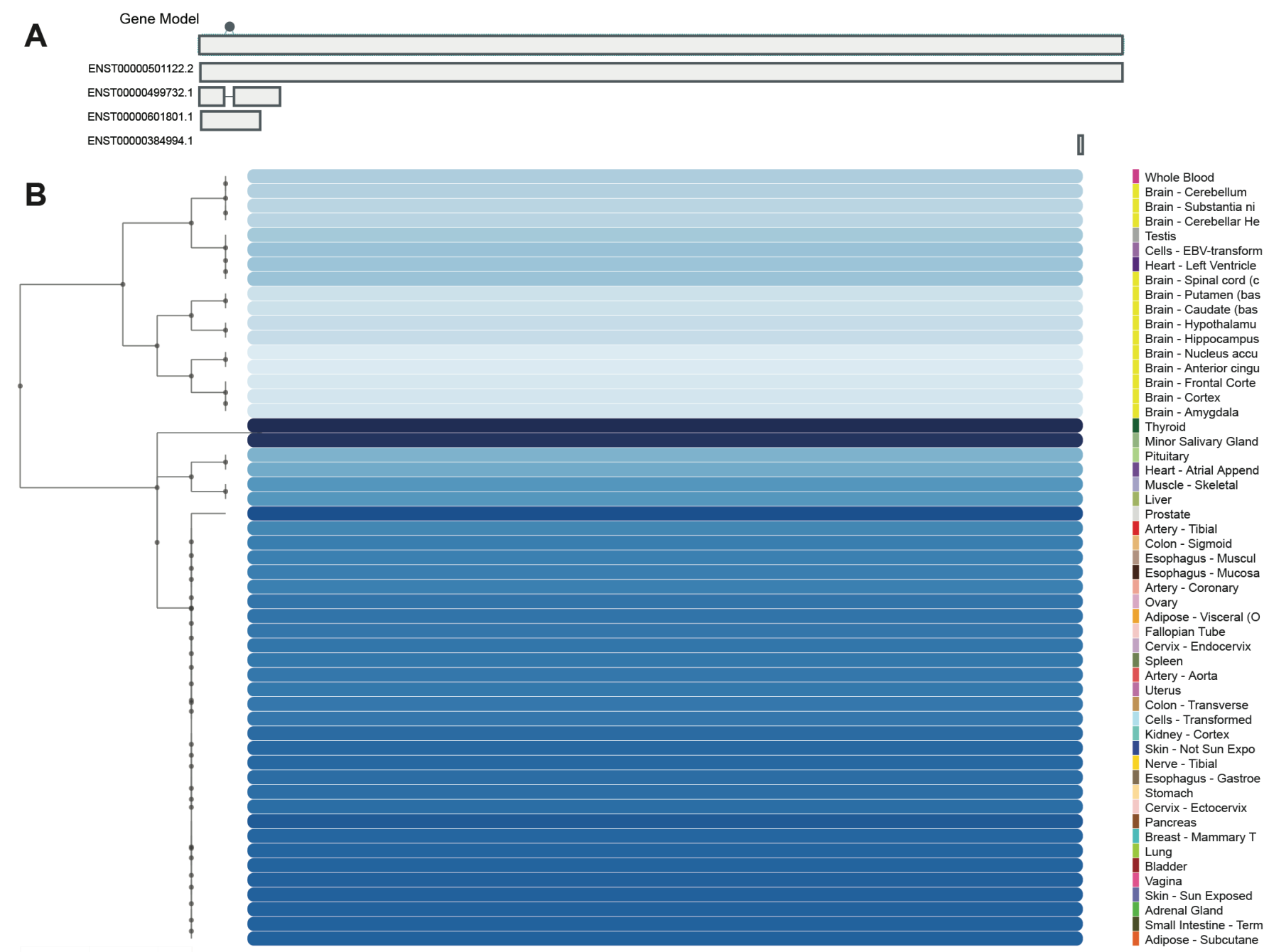

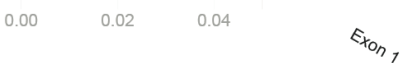

C

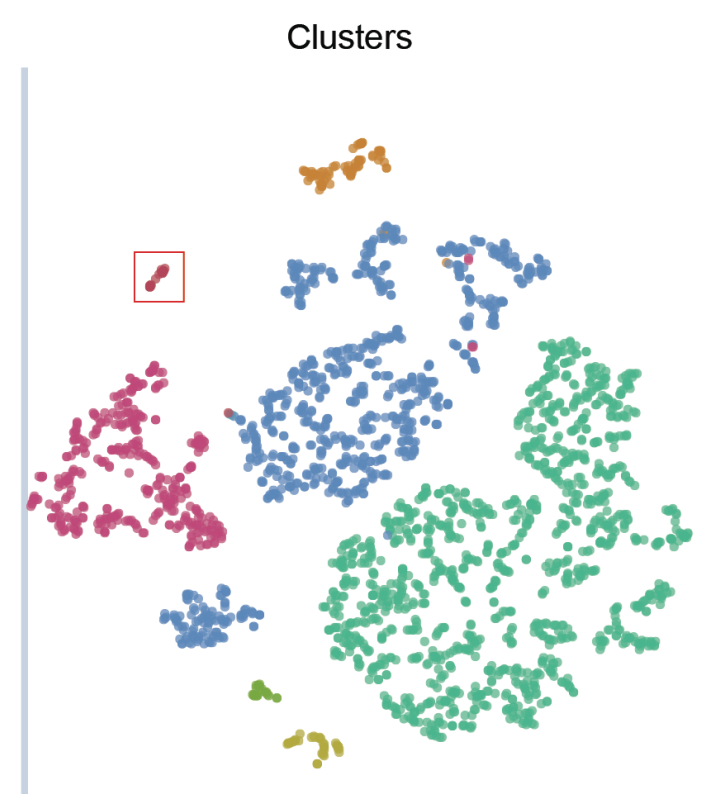

Clusters

- Astrocyte

- Neoplastic cell

- Oligodendrocyte

- Vascular cell
D

Neat1

Gene Expression

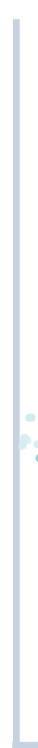

E

Malat1

Gene Expression

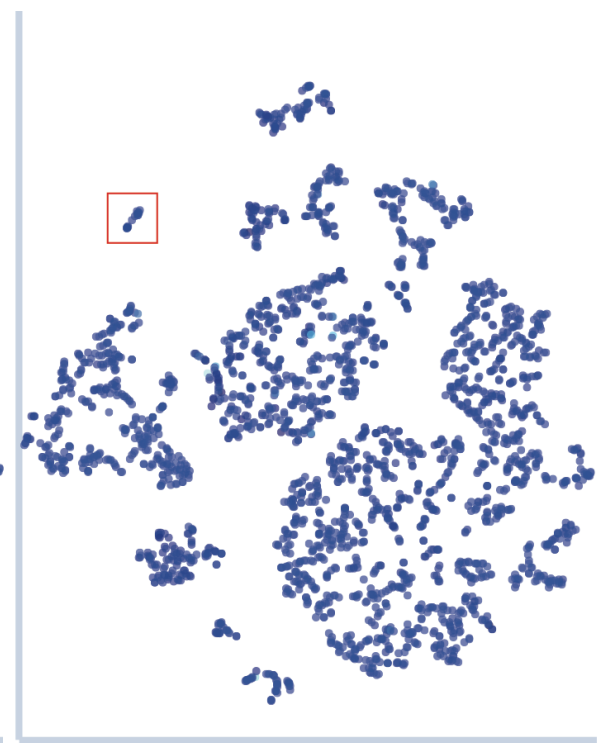


Supplemental Figure 1. NEAT1 expression is uniquely reduced in the human CNS, and baseline expression is low in human neurons relative to other cell types. (A) Diagram of NEAT1 gene structure used in hierarchical clustering of NEAT1 (B) Hierarchical clustering of NEAT1 based on exon level expression in 53 human tissues from the GTEx project. Dendrogram scale shows cluster distance. Expression values displayed in the heatmap are the median expression values in TPM for each exon in each tissue. (C-E) tSNE plots constructed using transcript per million (TPM) values from the transcriptomes of 3,589 biopsied human single cells. (C) Inferred cell types in unique colors, with clustered neurons outlined in red. (D) Expression of NEAT1 in single cells heatmap, from 0 to 1200 TPM. (E) Expression of MALAT1 (NEAT2) in single cells, from 0 to 12000 TPM. 

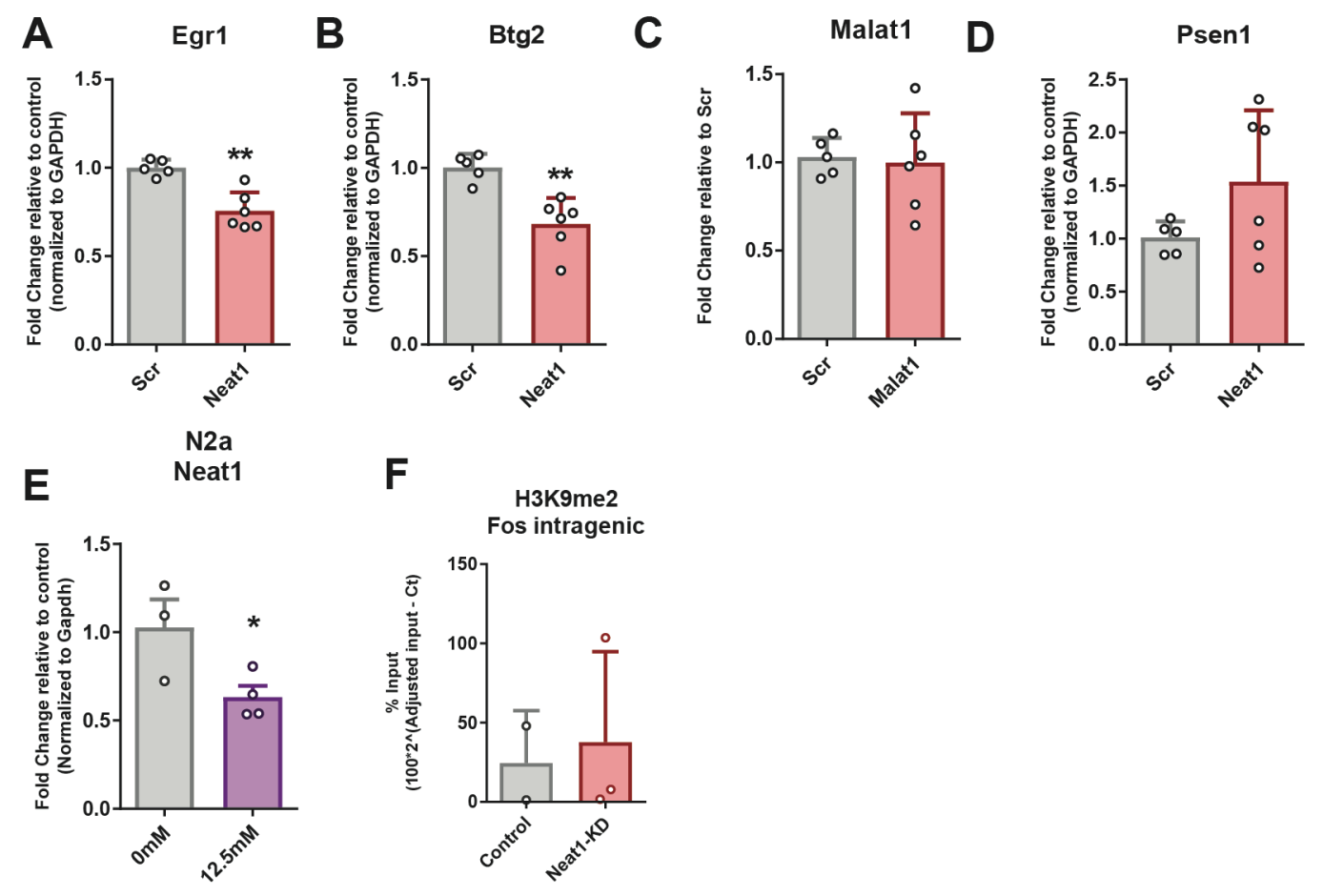

Supplemental Figure 2. Neuronal regulation of immediate early genes after Neat1 knockdown (A-D) Differential expression of the immediate early genes (A) Egr1 $(n=5,6 ; p=0.0010)$, (B) Btg2 $(n$ $=5,6 ; p=0.0018)$, (C) MALAT1 $(n=5,6 ; p=0.8133)$, and (D) Psen1 $(n=5,6 ; p=0.1228)$ after knockdown of Neat1 in cultured neuronal cells. (E) Expression of the IncRNA Neat1 in N2a cells after treatment with $\mathrm{KCl}(n=3,4 ; p=0.0495)(\mathrm{F}) \mathrm{H} 3 \mathrm{~K} 9 m e 2$ at an intragenic region of the $c$-Fos gene is unchanged after Neat1 knockdown in N2a cells $(n=2,3 ; p=0.7939)$. 
A
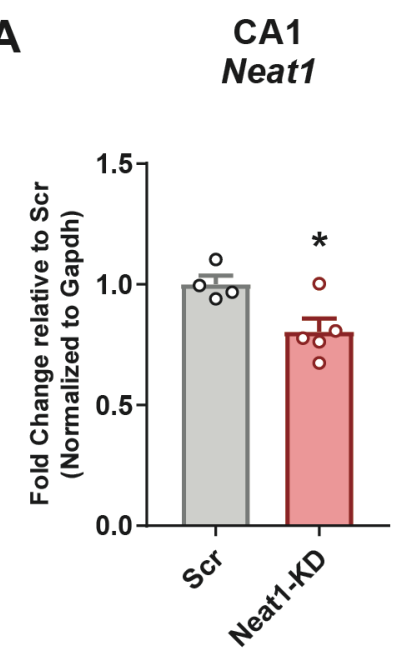

B

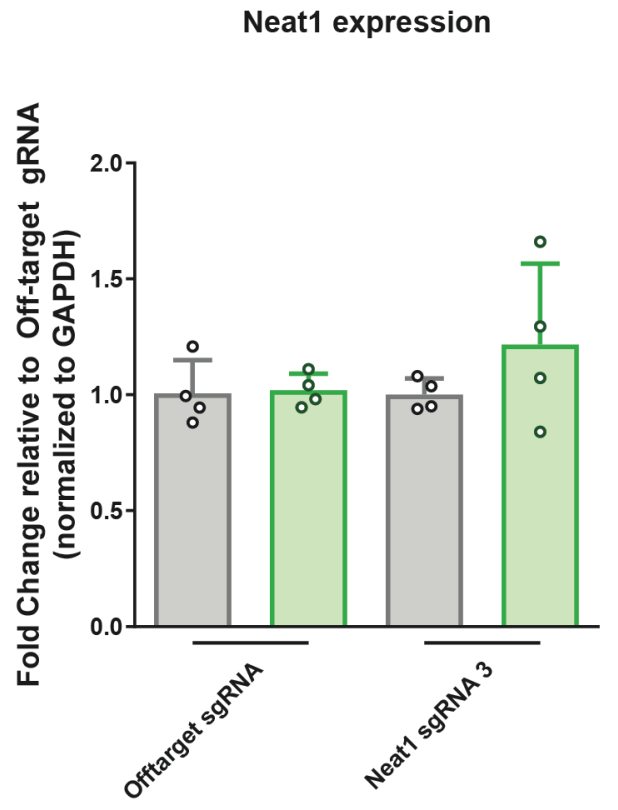

C

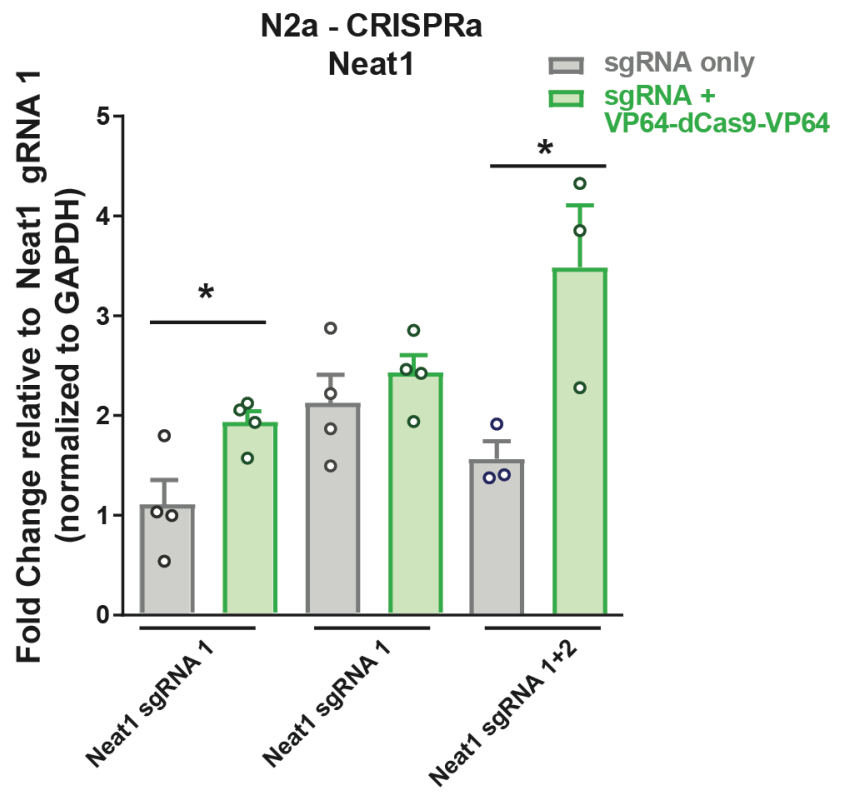

Supplemental Figure 3. Validation of Neat1 expression manipulation via RNAi and CRISPRa (A) RTQPCR quantification of Neat1 expression in dCA1 of naïve mice $5 \mathrm{~d}$ after in vivo transfection. $(n=4,5 ; p=$ 0.0242) (B-C) RT-qPCR quantification of Neat1 in response to three different sgRNAs for CRISPRa-mediated upregulation of Neat1. (B) Transfection of an off-target sgRNA plasmid alone, Neat1-targeting sgRNA 3 plasmid alone, or off-target sgRNA plasmid and ddCas9-2xVP64, while sgRNA 3 in conjunction with dCas9-2xVP64 had a modest effect on expression (not statistically significant; $p>0.05$ ). (C) Transfection of Neat1 sgRNA plasmids \#1 or \#1/\#2 in conjunction with dCas9-2xVP64 results in more robust transcriptional and additive upregulation of Neat1 relative to cells receiving only the respective $\operatorname{sgRNAs}\left(n=4,4,4,4,3,3 ;{ }^{*} p<\right.$ $0.05)$. 

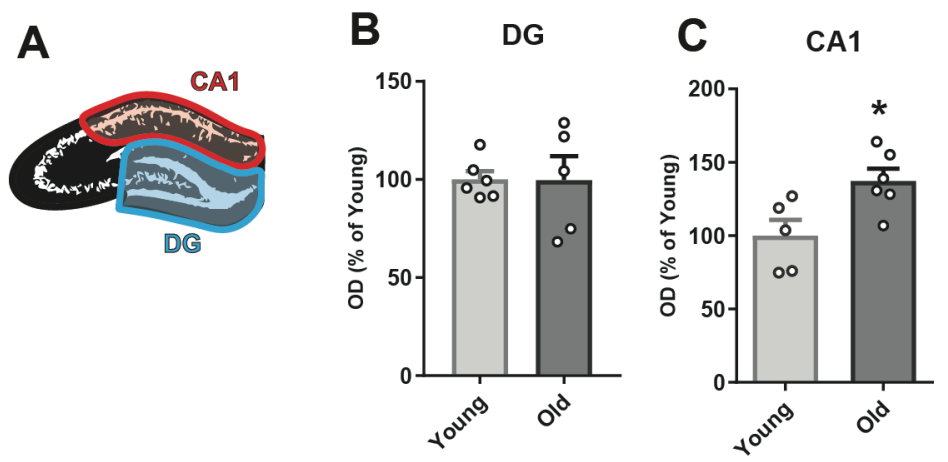

Supplemental Figure 4. Age-related elevation of H3K9me2 in dCA1 (A) Cartoon of dissection of CA1 from DG in Rattus norvegicus. (B) DG expression of H3K9me2 is unchanged between young and aged rats $(n=6,6 ; p=0.9762)$, while expression of H3K9me2 in CA1 (C) is elevated with aging $(n=5,6 ; p=0.0465)$. 
A

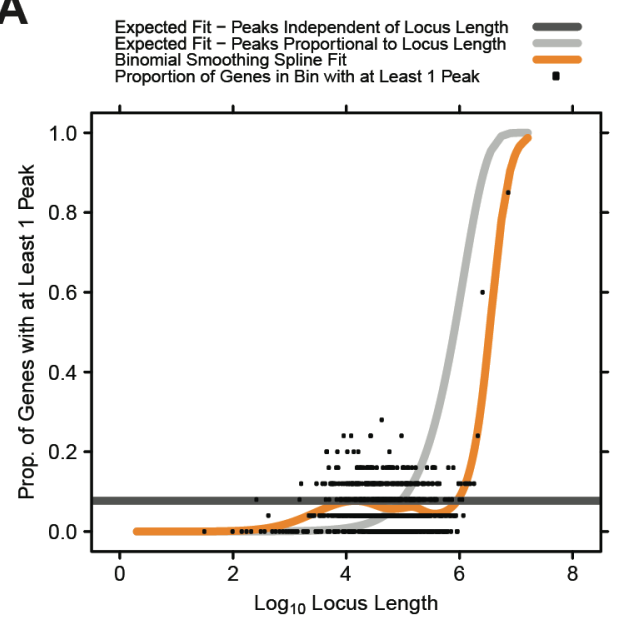

B

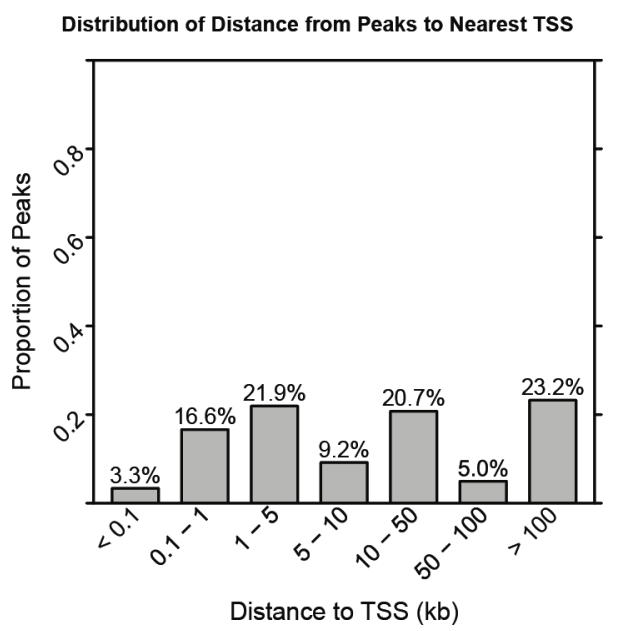

Supplemental Figure 5. Quality control plots from Chip-Enrich. (A) Curves comparing NEAT1-bound peaks to locus length. (B) Distribution of distance from NEAT1 bound peaks to the nearest gene TSS 